\title{
Introducing tomsup: Theory of Mind Simulations Using Python
}

Peter T. Waade ${ }^{*}, 1,2$, Kenneth C. Enevoldsen ${ }^{*}, 1,2,3$, Arnault-Quentin Vermillet ${ }^{2}$, Arndis Simonsen $^{2,4}$, and Riccardo Fusaroli ${ }^{1,2,5}$

*Equal contribution. Order decided by a tossed coin.

${ }^{1}$ School of Communication and Culture, Aarhus University

${ }^{2}$ The Interacting Minds Centre, Aarhus University

${ }^{3}$ Center for Humanities Computing Aarhus, Aarhus University

${ }^{4}$ Psychosis Research Unit, Aarhus University Hospital Psychiatry

${ }^{5}$ Department of Linguistics, Cognitive Science and Semiotics, Aarhus University

Affiliation

\section{Corresponding Authors}

Correspondence concerning this article should be addressed to either of the main authors:

Peter T. Waade (D) https://orcid.org/0000-0002-6061-0084

Email: peter@waade.net

Phone: +4520157606

Langelandsgade 139, 8000 Aarhus, Denmark

Kenneth C. Enevoldsen (iD https://orcid.org/0000-0001-8733-0966

Email: kenneth.enevoldsen@ cas.au.dk

Phone: +4520824404

Langelandsgade 139, 8000 Aarhus, Denmark 


\begin{abstract}
Theory of Mind (ToM) is considered crucial for understanding social-cognitive abilities and impairments. However, verbal theories of the mechanisms underlying ToM are often criticized as under-specified and mutually incompatible. This leads to measures of ToM being unreliable, to the extent that even canonical experimental tasks do not require representation of others' mental states. There have been attempts at making computational models of ToM, but these are not easily available for broad research application. In order to help meet these challenges, we here introduce the Python package tomsup: Theory of Mind Simulations Using Python. The package provides a computational eco-system for investigating and comparing computational models of hypothesized ToM mechanisms and for using them as experimental stimuli. The package notably includes an easy-to-use implementation of the variational recursive Bayesian $k$-ToM model developed by Devaine et al., 2014b and of simpler non-recursive decision models, for comparison. We provide a series of tutorials on how to: i) simulate agents relying on the $k$-ToM model and on a range of simpler types of mechanisms; ii) employ those agents to generate online experimental stimuli; iii) analyze the data generated in such experimental setup and iv) specify new custom ToM and heuristic cognitive models. Keywords: Theory of Mind, variational Bayesian inference, Game Theory, Agent-Based Simulation, Computational Modeling
\end{abstract}




\section{Introducing tomsup: Theory of Mind Simulations Using Python}

\section{Introduction}

Understanding what others believe and intend to do is crucial to navigate our everyday life. From figuring out how to pass each other in a narrow passage on a train to correctly identifying sarcastic comments in an email, we often have to infer each other's mental states in order to successfully interact. This ability is often called Theory of Mind (ToM) or simply mentalizing. Being able to measure how good we are at mentalizing has long been deemed crucial in order to assess social skills and impairments and more generally the ability to function in society (Bosacki \& Wilde Astington, 1999; Watson et al., 1999). However, there are still substantial controversies surrounding Theory of Mind (ToM): Which species display it? What role ToM deficits play in conditions such as autism? At what age infants develop it? (Bosacki \& Wilde Astington, 1999; Deschrijver \& Palmer, 2020; Kampis et al., 2020). At least part of the controversies are due to the fact that the notion of ToM is often under-specified and operationalized in heterogeneous ways, which makes comparison across studies difficult (Schaafsma et al., 2015). In order to advance more formal and systematic approaches to the definition and measure of ToM abilities, we introduce Theory of Mind Simulation using Python (tomsup), a Python package. tomsup is currently focused on k-ToM models (Devaine et al., 2014b) and simpler alternatives. More generally tomsup makes it easy to simulate agents with different ToM abilities in a variety of game-theoretical settings. This allows for testing formal ToM models in well-defined interactive contexts, and simplifies their implementation in experimental contexts. The tomsup package can be used to (1) explore the implications of formal ToM models, (2) develop empirical predictions and experimental paradigms, and (3) provide dynamical stimuli in experimental paradigms for testing ToM abilities. In the following, we briefly introduce ToM and the current discussion on the construct, as well as the computational approaches developed to formalize ToM; before delving into the details of the models implemented in tomsup and the usage of the package. ToM is a psychological theoretical construct describing the ability to correctly infer others' mental states. The notion was made popular by (Premack \& Woodruff, 1978) in their assessment of social-cognitive abilities in primates, and it quickly led to the development of 
the iconic false-belief tasks, assessing ToM in young children (Dennett, 1978; Wimmer \& Perner, 1983). This type of task usually consists of short stories where one of the characters develops a false belief (e.g. the character believes the cookie is hidden in the jar, while it's actually hidden in the drawer). While the participants know that the belief is false (e.g. they know the cookie is hidden in the drawer), they have to separate their own knowledge from what the character knows in order to correctly solve the task. ToM was thus initially conceived as the ability to correctly infer and represent other people's mental content independently of whether it mirrored one's own or not. This conception quickly led to the development of a large variety of operationalizations and experimental tasks: from inferring emotions in pictures of eyes and recorded speech, to inferring intentionality in videos of abstract forms moving around (e.g. Apperly, 2012, Quesque and Rossetti, 2020). ToM has thus become a cornerstone in the assessment of social abilities, especially in developmental disorders (Baron-Cohen, 2000; Baron-Cohen et al., 1985) and more recently in a wider range of mental disorders (Apperly, 2012; Berecz et al., 2016; Brüne, 2005). The notion of ToM and its operationalizations have, however, been widely criticised (Apperly, 2012; Boucher, 2012; Conway et al., 2019; Deschrijver \& Palmer, 2020; Quesque \& Rossetti, 2020; Schaafsma et al., 2015). First, it has been argued that ToM is too vague a construct. In other words, ToM is described only verbally, which leaves room for multiple interpretations and operationalizations of the concept. While this can be an advantage for early theory development and for maintaining multiple approaches, it can also lead to chronic and perhaps pathological under-specification. Indeed, it has been pointed out that different approaches and experimental paradigms, all purportedly assessing ToM, are conceptually incompatible, and often measure different cognitive processes than ToM. For instance, inferring emotions from faces, eyes or speech involves recognition of facial expressions, prosody, and the ability to linguistically express nuanced emotions. Analogously, inferring intentionality from the movement of abstract shapes requires kinematic discrimination and again linguistic abilities. See Bloom and German (2000), or Quesque and Rossetti (2020) for a more in depth discussion of the heterogeneous implementations of ToM. Second, it has been argued that the conceptual construct of ToM is not ecologically valid. The vast majority of the experimental tasks involve 
third person stances with little at stake: participants watch a video or hear a story and make an inference with no further consequences. These settings do not capture how people actually interact with each other, which may be a crucial component of how mentalizing unfolds. For instance, the increased emotional engagement, the need to react in real time and to anticipate the other's reactions - which form a crucial part of social interactions - may challenge the mentalizing system in a completely different way than the vast majority of experimental tasks (Dale et al., 2013; De Bruin et al., 2012; Tylen et al., 2012).

A lack of more precise mechanistic accounts with exact predictions prevents a clear understanding of how ToM abilities vary between individuals, how they apply to different contexts and how they relate to behaviors outside the lab. Indeed, recent research on mental disorders such as autism and schizophrenia shows that current operationalizations of ToM are not particularly effective at discriminating between patients and controls, or between different disorders; nor are they particularly informative as to actual levels of social functioning (De Bruin et al., 2012; Morrison et al., 2020; Pinkham et al., 2020; Sasson et al., 2020).

One venue to address the under-specification of the ToM construct is to develop computational models of ToM as a complementary strategy to current conceptual and experimental investigations. Having to develop a computational model of a cognitive ability forces researchers to specify their assumptions and make precise descriptions of the mechanisms at work, which in turn can lead to revisions of one's assumptions and ideas even before seeing any data (Devezer et al., 2019; Guest \& Martin, 2020; Smaldino, 2020; van Rooij \& Baggio, 2020). Computational models can then be compared using simulations or assessing their fit to behavioral data in order to critically revise them. Besides avoiding the issues of under-specification that is seen in much ToM research, theory-driven computational models of cognitive processes have been shown to provide more reliable estimates of individual differences, compared to standard statistical practices (such as ANOVAs), see Haines et al. (2020). Better estimates of individual differences are crucial for assessing whether ToM is involved in social impairments. Finally, recent work on computational models of ToM has included more interactive conceptions of ToM, which might help improve the ecological validity of the construct (Rusch et al., 2020). 
Notably, computational models related to ToM can be grouped into three categories that explicitly model others' mind to an increasing extent (Rusch et al., 2020). The first category consists of reinforcement learning based models, which are increasingly applied to social contexts. In these contexts an agent can observe the behavior of others and include that information in their own inferential and decision processes. These models are very effective at producing adaptive behaviour, but have no explicit modelling of others' mental states (Vélez \& Gweon, 2020). The second category consists of observational models (e.g. Baker et al. (2011)), which explicitly attempt to reconstruct others' beliefs based on a generative model of their mental states. However, observational models are limited in that others are represented as having mental states, but not as having a mentalizing system of their own. In other words, observational models cannot perform recursive ToM in which the agent infers not only what the other knows, but also what the other has likely inferred about the agent's own knowledge (and potentially further levels: what the other knows that the agent knows that the other knows, etc). The third category of models consists of recursive ToM models. These models take their roots in game theoretical settings where the actions of an agent are rewarded according to the action of the other agent. Therefore it is crucial for each agent not only to predict what the other is likely to do, but also the other's predictions as to the agent's own future behavior. Recursive ToM is, however, infamous for the computational load it imposes on agents: it is argued that humans can deal with three levels of recursion at the most, since representing many levels is a hard task, especially during an ongoing interaction where response speed is crucial (Camerer et al., 2004; Devaine et al., 2014a).

Several approaches have been suggested to tackle these issues. For example, Hampton et al. (2008) developed an 'influence' model, which uses Taylor decompositions to recursively estimate how much its actions affect the opponent's choice; while Yoshida et al. (2008) relied on optimal control theory to implement a Bayesian recursive ToM model. Inspired by these developments, Devaine et al. (2014a), Devaine et al. (2017), developed an approximate variational Bayesian approach, which forms the foundation for the work done in this paper. Variational Bayesian approaches provide fast to compute approximations of Bayesian inference by turning integration problems (hard to solve) into optimization ones (easier and 
faster to solve), thus providing realistic mechanisms through which complex Bayesian inferences could be implemented in cognitive systems. Recursive ToM models based on variational Bayes have been argued to be the most promising models so far: they are models of cognitive processes able to process information in real time; they are able to better anticipate and countermand other models' behaviors in game theoretical settings, plausibly thanks to the Bayesian use of prior information; and they provide useful predictions for testing human mentalizing abilities in interactive contexts (Rusch et al., 2020). Notably, Devaine et al. (2014a) explicitly compared these models with the influence models by Hampton et al. (2008) - the closest non-Bayesian equivalent - and found consistently equal or better performance. Indeed, recursive ToM models have been successfully - albeit exploratively deployed to better understand human and non-human primate ToM abilities in nuanced simulation and experimental setups. Conjoined simulation and experimental studies have explored how many levels of recursion (the agent representing another agent representing the former, etc.) would be meaningful given the specifications of the model (Devaine et al., 2014a, 2014b). The studies show that the models have a hard time effectively reconstructing the recursion of ToM beyond three levels (I infer that you infer that I infer that you infer). In other words, attempting to infer additional levels of ToM results in representations so uncertain that it is impossible to discriminate them from models only including 3 levels. The models do better at identifying how many levels of ToM should be employed when in competitive settings, compared to cooperative ones. This turned out to be largely in accord with empirical human data (Devaine et al., 2014a, 2014b). Crucially, the models were able to effectively assess differences in ToM abilities in clinical populations characterized by social impairments (D’Arc et al., 2018), and across primate species (Devaine et al., 2017), which bodes well for investigating further applications.

Accordingly, we chose to focus on these variational recursive ToM models in the tomsup package. They provide the currently most promising formal and precise description of how agents might represent other agents' minds, thus answering the critique of an under-specified ToM construct. They have also been developed to model ToM in interactive situations, thus at least partially answering the critique to the lack of ecological validity of the ToM construct. 
Finally, they have shown promise in being realistic models of actual cognitive processes, in developing testable predictions and in assessing individual and group-level differences in social skills (D’Arc et al., 2018; Devaine et al., 2014a, 2014b). The tomsup Python package aims to build on these promising models by implementing them in a general open-source framework for agent-based simulations and game theoretic experiments relating to ToM. The game theoretic setting is formally well-defined and allows for testing empirical hypotheses in an interactive context, under simple assumptions. Game Theory has shown usefulness in the analysis of a variety of real-world phenomena, such as climate change (DeCanio \& Fremstad, 2013), coordination (Devaine et al., 2014a) and other behavioural contexts (Camerer, 2010). Indeed, the tomsup package provides not only a formal implementation of variational recursive ToM in interactive contexts, but it also provides the tools to understand the consequences of this model. The model is implemented in the context of game theoretical settings of repeated decision making, such as the matching pennies game (see section 2 . The tomsup Package for details). The package includes alternative mechanisms to ToM (that is, to the explicit representation of others' state of mind), such as heuristic strategies (keep choosing the same if you win, change choice otherwise) and reinforcement learning. The package enables the user to set up simulated interactions between agents relying on these different computational models (different cognitive mechanisms). Thus, the user can simulate data in diverse contexts to explore the implications of the ToM model, and identify interesting cases to be tested empirically.

It should be noted that an implementation of variational recursive ToM models is available in the variational Bayesian analysis tool, VBA toolbox for MATLAB (Daunizeau et al., 2014; Devaine et al., 2017). The VBA toolbox and tomsup are complementary in many ways and tomsup does not aim at replacing the full functionalities of VBA (which is a more general variational inference software). The k-ToM implementations provided by tomsup and VBA display exact behavioral consistency, i.e. they produce the same inferences and behaviors; and tomsup is currently computationally more efficient (see Appendix $\mathrm{C}$ for a series of tests). Crucially, tomsup is not only open-source, but free to use; while VBA - relying on the MATLAB programming language - requires a MATLAB license to run. Therefore, the tomsup 
package for Python facilitates a wider access to the models across research communities, as well as an easier integration in a variety of open source tools (e.g. online experiments) that rely on more widespread free languages such as $\mathrm{R}$ and Python. Not least, tomsup is developed specifically with usability in mind, in order to facilitate further development and use at a more accessible technical level. Furthermore, tomsup is centered around research on ToM rather than variational inference in general. This provides a focus on application of models in varying simulated and experimental settings, supporting model interpretability and transparency, and allowing implementation of - and comparison with - new proposed ToM models. On the other hand, VBA provides a broader suite of tools, including model fitting and comparison to analyze empirical data. So the package choice should be based on the research questions being asked.

In the following paragraphs we will first introduce the basic setup of the tomsup package, then proceed to explain the specific computational model of ToM that is included in tomsup (based on Devaine et al. (2014a)), and the computational models of plausible alternative cognitive mechanisms, such as reinforcement learning and heuristic methods. We then explain how to use tomsup for simulating tournaments or for making simulated agents interact with participants, followed by an experimental use case and a validation of the recursive k-ToM model. Finally, we present a case simulation study where ToM agents of different sophistication levels compete with each other and agents with a variety of simpler strategies. We also show how to use tomsup to let participants play against ToM agents in an experimental context.

\section{The tomsup Package}

tomsup is a Python package implementing recursive ToM models within a larger agent-based modeling framework in a game theoretic context. Within tomsup the user can easily explore the implications of computational models of ToM by implementing them in agents competing in different formalized interactions (games) - e.g. the prisoner's dilemma, the stag-hunt and the matching pennies games - and assessing their performance in different conditions. Agents can be endowed with different cognitive mechanisms and strategies and compete in diverse 
environments to better understand the resulting behaviors and their relative advantages and disadvantages. Crucially, tomsup can also be used to dynamically generate stimuli for experimental setups, that is, having agents with different cognitive mechanisms interact in real time with human participants. This enables the user to assess how participants could adapt to different strategies, and which of the implemented mechanisms best match the participants' behaviour. Further, the agents can be used to infer which levels of recursion and other parameters participants might be using.

tomsup implements a variety of economic games. Each game implemented involves repeated 2-agent interactions in which the agents synchronously choose between two possible choices, and the payoff they get for their choice depends on what the other agent has chosen. Indeed, the framework generalizes to any 2-player scenario that can be operationalized as a 2-by-2 payoff matrix. In a game theoretic context, a payoff matrix is a mathematical abstraction of real-life situations attempting to represent the possible outcomes of the situation in terms of the choices taken and rewards or punishments that follow. Let us consider the example of the competitive matching pennies game, a prime case for the application of ToM (Devaine et al., 2017). The matching pennies game represents a situation where one agent (or participant) has to hide a coin in one hand, and the other agent has to guess which hand. The situation, in which if the second agent guesses correctly, the first loses (see Table 1), is a schematic representation of all situations in which a person's loss is directly proportional to another person's gain. Crucially, this game can only be consistently won by predicting on a turn by turn basis the opponent's choice (and therefore ToM becomes relevant).

In order to enact decisions in these games, the agents rely on computational implementations of decision processes (or cognitive mechanisms). tomsup includes a variety of such models (or game theoretic agents). The key model is the ToM model at different levels of recursion (explained in section 2.1. The $k$-ToM Model). However, simpler models have been implemented as plausible alternative mechanisms to fully fledged ToM models. Some are heuristic strategies, that is, simple rules. The simplest is the Random Bias agent, which simply makes a random choice with a given probability (e.g. 60 percent probability of choosing the right hand in the matching pennies game). Tit-For-Tat follows the choice employed by the 


\section{Table 1}

The competitive matching pennies game. Each cell denotes the reward for player 1 and player 2 for example if player 1 has put the penny in the right hand, and player 2 chooses the right hand, player 1 would lose 1 point (-1) and player 2 would gain $1(+1)$.

Matching Pennies Player 2

\begin{tabular}{ll|l|l|} 
& & \multicolumn{1}{c}{ Right } & \multicolumn{1}{c}{ Left } \\
\cline { 3 - 4 } Player 1 & Right & $-1,1$ & $1,-1$ \\
\cline { 3 - 4 } & Left & $1,-1$ & $-1,1$ \\
\cline { 3 - 4 } & & &
\end{tabular}

other agent in the previous interaction. Win-Stay Lose-Shift bases its decisions on the previous round: if the agent won, it keeps choosing the same, while if it lost, it changes its decision (Axelrod \& Hamilton, 1981). Other models implement more complex strategies, for instance using statistical learning strategies to infer statistical regularities in the environment as opposed to explicit modeling of other's mental states. We implemented a reinforcement learning model (a Q-Learning agent, as proposed by Watkins and Dayan (1992)). Such agents update their expectations of rewards for each choice on a turn by turn basis, according to the previous rewards achieved.

In tomsup, the agents' interactions and environments can be structured in many ways. In the simplest case, two agents interact repeatedly with each other. However, multiple agents can be involved, e.g. in round robin tournaments where each of multiple agents competes against each of the others. Further, more realistic scenarios with multiple agents can be played out on networks where who competes against whom is determined by the network structure (e.g. to simulate the effects of agents only competing with their local neighbors). The effects of diverse "environments" for the interactions has been previously used for instance to examine neighborhood segregation (Schelling, 1978) and cooperation (Axelrod \& Hamilton, 1981).

After having introduced the general structure of the interactions, we now focus on the recursive ToM implementation. 


\subsection{The $k$-ToM Model}

In this section we first cover a conceptual overview of the recursive ToM model developed throughout Devaine et al. (2014b), Devaine et al. (2014a), and Devaine et al. (2017). We then focus on the mathematical implementation of such recursive ToM agent (from now on k-ToM agents, where $\mathrm{k}$ is an index of how many levels of recursion/sophistication are implemented). Note that where our equations differ from the original articles, this is simply due to our decision to be more transparent as to the approximations implemented, which are the same for both VBA and tomsup. All ToM agents attempt to predict the probability of their opponents' possible choices. This enables the agents to calculate the expected reward for each of their own possible choices, so to choose the one with the highest expected reward (for more details see section The Decision Process). A key element of ToM agents is the way in which they represent their opponents in order to predict their choices. 0-ToM agents $(\mathrm{k}=0$, i.e., no recursion) conceive of their opponents as biased agents, that is, with a given probability for each choice. $k$-ToM agents with a $k$ above zero, on the other hand, treat their opponents as ToM agents, that is, as agents with internal representations. In other words, these k-ToM agents actually simulate their opponents' learning process, to be able to infer the opponent's beliefs about themselves. $k$ indicates how many layers of recursion the agent can represent, that is, at which level it stops representing the opponent as representing the agent as representing the opponent, etc. 1-ToM represents the opponent as a 0-ToM agent; 2-ToM can represent the other agent as a 1-ToM or a 0-ToM agent; and so on. Crucially, a k-ToM agent must also infer the level $\mathrm{k}$ of its opponent from the options $[0 ; k-1]$, which is done in a reinforcement learning-like fashion as described in section $k$-ToM's Learning Process. The ToM model consists of a learning process where it infers the opponent's internal parameters and estimates the probability of the opponent choosing either option (see the sections 0-ToM's Learning Process and $k$-ToM's Learning Process); and a decision process where the expected choice of the opponent is used to probabilistically define the agent's choice (see the section The Decision Process). All k-ToM agents are characterized by four parameters collectively referred to as $\theta$. The behavioural temperature $\beta \in] 0 ; \infty[$ indicates how noisy the decision process is, that is, how strongly determined the decision is by the predicted choices of the 
opponent. The volatility $\sigma \in] 0 ; \infty[$ indicates how much the agent thinks that the opponent might shift their parameters over time (and accordingly controls updating and confidence in the agent's parameters). The bias $b \in]-\infty ; \infty[$ indicates a preference towards a certain choice independent of all other parameters (e.g. right handed participants might prefer to choose the option on the right). Finally, the dilution $d \in[0 ; 1]$ indicates the degree to which beliefs about the opponent's sophistication level are forgotten over time (thus giving more or less weight to more recent interactions), which can be interpreted as an assumed base rate of change in the opponent's sophistication level. Note that if simpler models are necessary and/or warranted, both the dilution $d$ and the bias $b$ are optional parameters in tomsup, that is, they can be set to have no role in the agent's and/or opponent's learning and decision processes.

\section{The Decision Process}

Given the inferred representation of the opponent (see section 0-ToM's Learning Process and $k$-ToM's Learning Process for details on the inference), on each trial the agent produces an estimate of the probability that the opponent will choose $1 p_{t}^{o p}$. The expected payoff of choosing 1 relative to $0 \Delta V_{t}$ is then calculated on each trial $t$ by weighting the relative payoff of each choice given the probability of the opponent's choice of 1 according to the following equation:

$$
\Delta V_{t}^{\prime}=p_{t}^{o p}(U(1,1)-U(0,1))+\left(1-p_{t}^{o p}\right)(U(1,0)-U(0,0))
$$

The notation $U\left(c^{\text {self }}, c^{o p}\right)$ denotes the payoff (utility) function, which returns the reward $R$ given a payoff matrix and the (hypothetical) choices of $k$-ToM agent $c^{\text {self }}$ and the opponent $c^{o p}$.

After this, the optional bias parameter $b$ can be added to bias the expected utility towards a specific choice:

$$
\Delta V_{t}=\Delta V_{t}^{\prime}+b
$$

Where $\Delta V_{t}^{\prime}$ is the value of $\Delta V_{t}$ before the optional update. The bias parameter $b$ is useful to model agents or estimate opponents which might present an increased tendency to choose one 
option over the other (everything else being equal), for instance opponents implementing a Random Bias strategy, or right handed participants tending to favor the option on the right more than their objective utility function would predict.

Then k-ToM's own decision probability $P\left(c_{t}^{\text {self }}=1\right)$ is calculated by inserting the expected utilities $\Delta V_{t}$ in a softmax decision rule for two options, as shown below:

$$
P\left(c_{t}^{\text {self }}=1\right)=\frac{1}{1+\exp \left(-\frac{\Delta V_{t}}{\beta}\right)}
$$

Where $P\left(c_{t}^{\text {self }}=1\right)$ is $k$-ToM's probability of choosing 1 on the current trial $t$. $\beta$ denotes 0-ToM's behavioural temperature parameter, where higher values lead to more random behaviour.

\section{0-ToM's Learning Process}

Figure 1 graphically represents the 0-ToM model. 0-ToM assumes that the opponent uses a Random Bias strategy. In other words, the learning process for 0-ToM agents consists in using the previous trial to estimate the bias of the opponent $\left(p_{t}^{o p}\right)$ towards a given choice. The mean $\mu$ and variance $\Sigma$ of the bias are estimated and then combined into a point estimate to simplify the decision process (see section The Decision Process).

The rest of this section details the mathematical implementation of the learning process. First, the variance $\Sigma$ of the estimate of the opponent's bias is updated using the following equation:

$$
\Sigma_{t} \approx \frac{1}{\frac{1}{\Sigma_{t-1}+\sigma}+s\left(\mu_{t-1}\right)\left(1-s\left(\mu_{t-1}\right)\right)}
$$

Where $\Sigma_{t}$ denotes the variance or uncertainty of 0-ToM's estimate of the opponent's bias at trial $t$, and $\mu_{t-1}$ is the mean in log-odds of the estimate from the previous trial. $s$ is the sigmoid function used to convert the mean parameter estimate into a probability between 0 and 1. $\sigma$ denotes the 0-ToM agent's volatility parameter, which is an assumption on how much the opponent's parameters vary across time, defining a lower bound for how certain the 0-ToM agent can be of their opponent's bias estimate. 


\section{Figure 1}

A graphical model of the 0-ToM model's learning and decision processes, which is repeated on each trial. Variables that are observed (data) are shaded. Unobserved deterministic variables are represented with a double border. Discrete variables are represented as squares, while continuous ones as circles. Note that the optional update of $\Delta V$ has been omitted for simplicity.

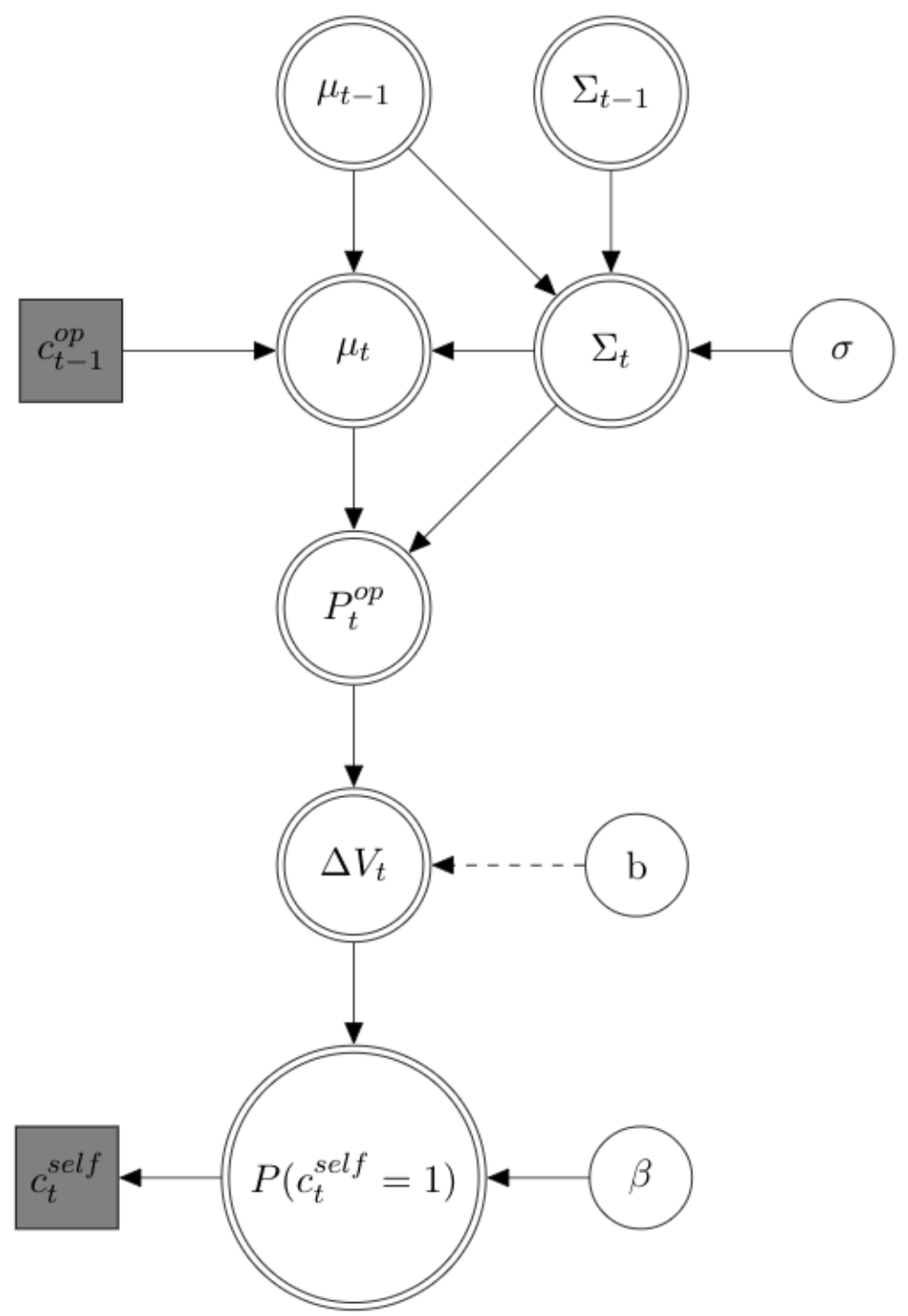


The updated $\Sigma$ is then used when updating the mean $\mu$ estimate of the opponent's bias, as shown below:

$$
\mu_{t} \approx \mu_{t-1}+\Sigma_{t}\left(c_{t-1}^{o p}-s\left(\mu_{t-1}\right)\right)
$$

Here $c_{t-1}^{o p}$ denotes the opponent's choice at the previous trial $t-1$. The $\mu$ estimate is updated based on the difference between the opponent's actual choice $c_{t-1}^{o p}$ and the estimated choice probability $s\left(\mu_{t-1}\right)$ on the last trial (i.e. the prediction error), weighted by the uncertainty $\Sigma$ (the more uncertain the current estimate, the more the error in the estimate from the previous trial will change it). Equation 4 and Equation 5 together form the equivalent of a Kalman filter and are used to approximate opponent's choice probability.

In order to calculate a point estimate of the opponent's bias, that is, the probability that the opponent will choose 1 in this trial, the mean $\mu$ and variance $\Sigma$ of the estimate are combined according to the following equation:

$$
p_{t}^{o p} \approx s\left(\frac{\mu_{t}}{\sqrt{1+\left(\Sigma_{t}+\sigma\right) 3 / \pi^{2}}}\right)
$$

$p_{t}^{o p}$ is the estimated probability of the opponent choosing 1. $\mu$ and $\Sigma$ are the mean and the variance for the opponent's bias. $\sigma$ is the volatility parameter (estimated tendency of the opponent to change its bias over time). The equation implies that the higher the uncertainty in the estimate, the more the estimated probability of the opponent choosing 1 is pulled towards chance level and away from the mean $\mu$. To avoid identifiability issues Daunizeau et al. (2014) approximate the equation to:

$$
p_{t}^{o p} \approx s\left(\frac{\mu_{t}}{\sqrt{1+0.36 \cdot \Sigma_{t}}}\right)
$$

The tomsup package implements both variants with the approximation as a default. $p_{t}^{o p}$ is then used in the decision process as described in section The Decision Process.

\section{k-ToM's Learning Process}

When agents implement a more sophisticated representation of the opponent, that is, when $\mathrm{k}>$ 0 , the learning process becomes more complex, as shown in Figure 2. $k$-ToM agents with 
$k>0$ simulate the opponent's learning and decision processes in order to estimate its probability of choosing $1, p^{o p}$. The opponent is represented as a $k$-ToM agent of a lower sophistication level $\kappa<k$. The agent, thus, has to estimate on a trial by trial basis the probability $\lambda^{\kappa}$ of the opponent having each of the possible levels $\kappa$, besides their model parameters $\theta$. Given that the model is recursive (representing the opponent representing the agent representing...), estimates of the opponent's parameters also involve estimating the opponent's estimation of the agent's own parameters. This is done using a non-linear variational Bayes Laplace approximation, yielding a mean estimate $\mu^{\theta}$ and variance $\Sigma^{\theta}$ for each of the opponent's parameters included in $\theta$ (the behavioural temperature $\beta$ and the volatility $\sigma$, and optionally the bias $b$ and the dilution $d$ ). Using these estimates, the k-ToM agent simulates the opponent's beliefs about its own choice probability, and consequently infers the opponent's choice probability $\mu$ of choosing 1 . During this process, the gradient $W^{\theta}$ is defined as the change in estimated choice probability of the opponent $p^{o p, \kappa}$ due to the change in parameter estimates $\mu^{\kappa, \theta}$, separately for each parameter in $\theta$. The gradient $W^{\theta}$ later determines how much parameter estimates $\mu^{\kappa, \theta}$ are updated so that less important parameters are updated less; and weighs $\Sigma^{\kappa, \theta}$ so that uncertainties about more important parameters have greater effect when forming beliefs about the opponent's choice probability $p^{o p, \kappa}$. Parameters and choice probability are estimated for each of the opponent's possible levels of sophistication $\kappa<k$. The final estimated probability for the opponent to choose $1 p^{o p}$ is the weighted average of the probabilities of choosing 1 for each possible opponent level $p^{o p, \kappa}$, where the weight is defined by the probability $\lambda^{\kappa}$ of the opponent using that level of recursion. This final estimate is then used in the agent's decision process in the same way as for the simpler 0-ToM agent, described in The Decision Process. 


\section{Figure 2}

A graphical model of the k-ToM model's learning and decision processes, which is repeated on each trial. Variables that are observed (data) are shaded. Unobserved deterministic variables are represented with a double border. Discrete variables are represented as squares, while continuous ones as circles. Note that the optional updates of $\Delta V$ and $\lambda^{\kappa}$ have been omitted for simplicity.

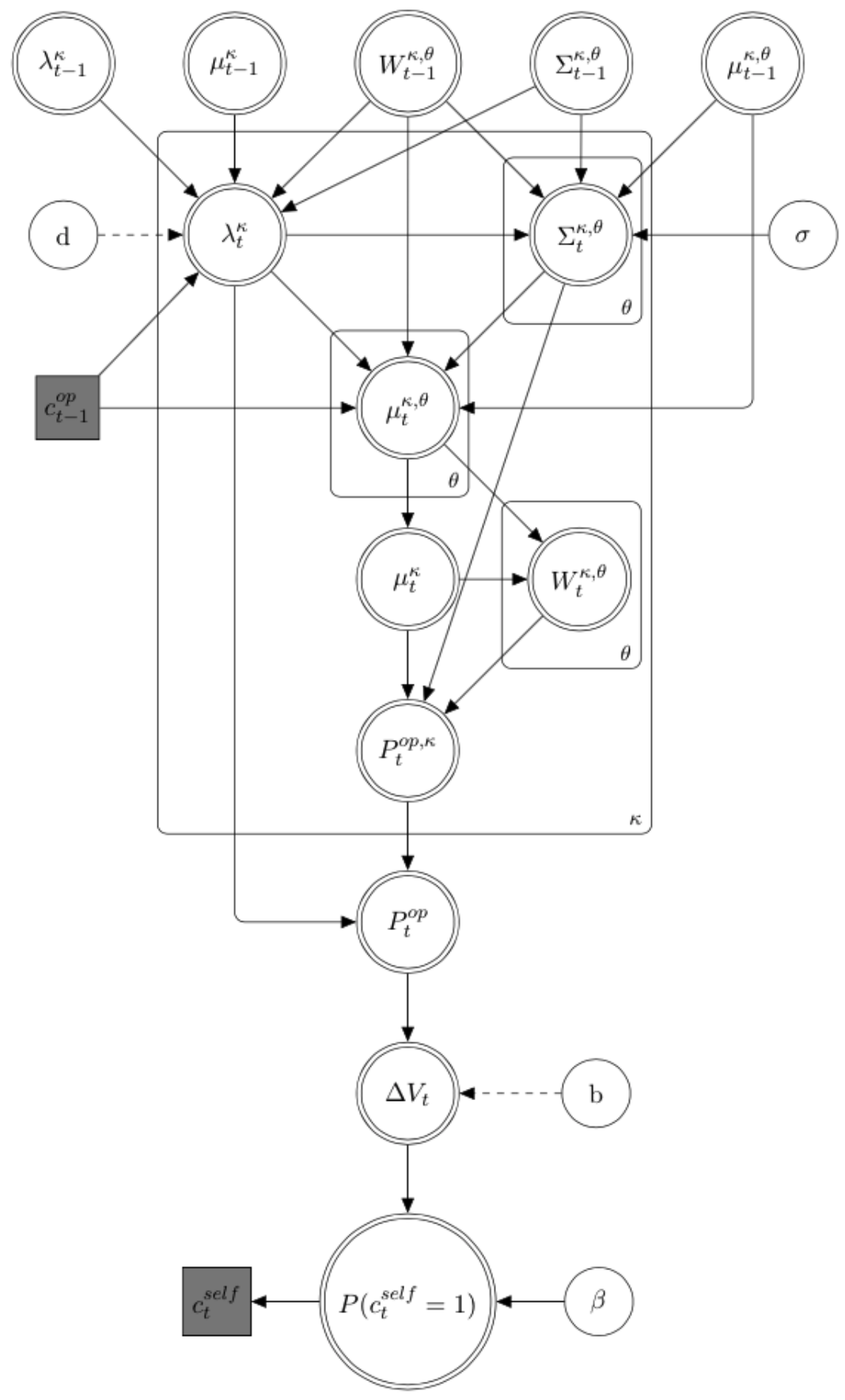


Here follows the mathematical implementation. First the agent estimates the probability $\lambda^{\kappa}$ that the opponent has each of the possible sophistication levels $\kappa$. The dilution parameter $d$, if used, increases the uncertainty of $\lambda^{\kappa}$ estimates from the previous trial ('forgetting' them) to facilitate inferring changing opponent's parameters. This is shown in the following equation:

$$
\lambda_{t-1}^{\kappa}=(1-d) \cdot \lambda_{t-1}^{\prime \kappa}+\frac{d}{k}
$$

Where $\lambda_{t-1}^{\prime \kappa}$ is the value of $\lambda_{t-1}^{\kappa}$ before the optional update. $d$ is the dilution parameter (on a $0-1$ probability scale, as transformed by the sigmoid function), and $k$ is $k$-ToM's sophistication level, incidentally also equal to the amount of possible levels of recursion in the opponent.

Probability estimates for each recursion level are updated by comparing the expected behavior under each possible opponent level to the observed behavior:

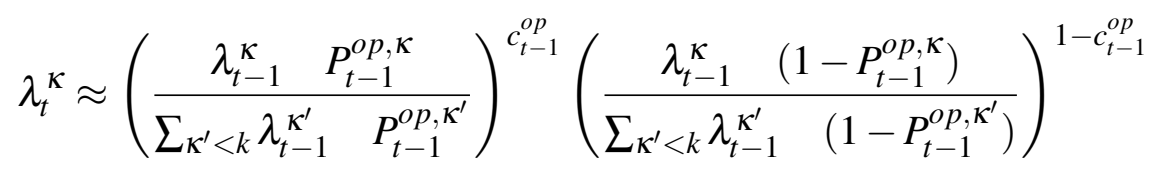

Here $\lambda_{t-1}^{\kappa}$ denotes the estimated probability $\lambda$ at trial $t$ of its opponent having a sophistication level of $\kappa \cdot p_{t-1}^{o p, \kappa}$ denotes the estimated probability for the opponent to choose 1 for each possible opponent level $\kappa$ on the previous trial $t-1$. Note that $\Sigma$ is here used as a summation sign, and not to denote parameter estimate uncertainties. This is done to keep the notation consistent with the notation used by Devaine et al. (2017).

Following Daunizeau et al. (2014), $P_{t-1}^{o p, \kappa}$ is approximated using the following equation:

$$
P_{t-1}^{o p, \kappa} \approx s\left(\frac{\mu_{t-1}^{\kappa}-0.319 \cdot\left(\Sigma_{t-1}^{\kappa}\right)^{0.781}}{\sqrt{1+0.205 \cdot\left(\Sigma_{t-1}^{\kappa}\right)^{0.870}}}\right)
$$

Here $\mu_{t-1}^{\kappa}$ is the (log-odds) probability for the opponent to choose 1 at trial $t$, predicted from the previous trial $t-1$, for each possible opponent level $\kappa . \Sigma_{t-1}^{\kappa}$ is the agent's uncertainty about that choice probability estimate, which is an average of the uncertainties about the agent's parameter estimates weighted by their influence on estimates of behavior:

$$
\Sigma_{t-1}^{\kappa} \approx \sum_{\theta} \Sigma_{t-1}^{\kappa, \theta}\left(W_{t-1}^{\kappa, \theta}\right)^{2}
$$


Here $\Sigma_{t-1}^{\kappa, \theta}$ denotes the agent's uncertainty as estimated in the previous trial $t-1$ for each parameter $\theta$ and each possible recursion level $\kappa . W_{t-1}^{\kappa, \theta}$ is the gradient of the effect of parameter estimates on choice probability estimates. Note that this equation only is valid under a mean-field approximation among parameters.

The k-ToM agent now updates its estimates for each of the opponent's parameter values $\theta$ (the behavioural temperature $\beta$ and the volatility $\sigma$, and optionally the bias $b$ and the dilution $d$ ). First the uncertainty of the parameter estimates is calculated:

$$
\Sigma_{t}^{\kappa, \theta} \approx \frac{1}{\frac{1}{\Sigma_{t-1}^{\kappa, \theta}+\sigma}+s\left(\mu_{t-1}^{\kappa}\right)\left(1-s\left(\mu_{t-1}^{\kappa}\right)\right) \lambda_{t}^{\kappa}\left(W_{t-1}^{\kappa, \theta}\right)^{2}}
$$

Here $\mu_{t-1}^{\kappa}$ is the agent's estimate the opponent's probability of choosing 1 , for each possible opponent level $\kappa .^{1}$

The mean estimates of each parameter in $\theta$ the are now updated:

$$
\mu_{t}^{\kappa, \theta} \approx \mu_{t-1}^{\kappa, \theta}+W_{t-1}^{\kappa, \theta} \Sigma_{t}^{\kappa, \theta} \lambda_{t}^{\kappa}\left(c_{t-1}^{o p}-s\left(\mu_{t-1}^{\kappa}\right)\right)
$$

$\mu^{\kappa, \theta}$ is updated according to the difference between the observed behaviour $c_{t-1}^{o p}$ and the choice probability $s\left(\mu_{t-1}^{\kappa}\right)$ (i.e. a prediction error), weighted by the gradient of the effect of parameter estimates on choice probability estimates $W^{\kappa, \theta}$, the probability of the opponent having the given sophistication level $\lambda^{\kappa, \theta}$, and the uncertainty of the estimate $\Sigma^{\kappa, \theta}$. The agent now calculates the mean expected probability $\mu^{\kappa}$ for the opponent's choice, given each possible level of recursion $\kappa$, by simulating the opponent's learning process. This includes storing and updating the beliefs of the simulated opponents of the different levels $\kappa$. The agent then numerically estimates the gradient $W$ of the effect of parameter estimates $\mu^{\theta}$ on choice probability estimates $\mu$ fr each possible opponent level $\kappa$ :

$$
W_{t}^{\kappa, \theta} \approx \frac{d \mu_{t}^{\kappa}}{d \mu_{t}^{\kappa, \theta}}
$$

\footnotetext{
${ }^{1}$ Note that, as in the VBA package for MATLAB (Daunizeau et al., 2014), the volatility $\sigma$ is set to 0 when estimating the opponent's behavioural temperature $\beta$, as it simplifies the computation.
} 
The approximation is done by a local linearization, finding the difference made to choice probability estimates $\mu$ by a small increment in each parameter estimates $\mu^{\theta}$. This is done for each parameter included in $\theta$ (the behavioural temperature $\beta$ and the volatility $\sigma$, and optionally the bias $b$ and the dilution $d$ ). $W^{\theta}$ is necessary for ensuring that parameter estimates are updated and weighed appropriately because they have a non-linear relation to the observed behavior of the opponent.

Next, the agent estimates the opponent's probability of choosing 1 (using an approximation to avoid unidentifiability issues, as in Equation 7) for each of the possible levels $\kappa$, taking uncertainty into account:

$$
p_{t}^{o p, \kappa} \approx s\left(\frac{\mu_{t}^{\kappa}}{\sqrt{1+0.36 \cdot \Sigma_{t}^{\kappa}}}\right)
$$

Where $\mu_{t}^{\kappa}$ is the mean estimate of the opponent's probability of choosing 1 on trial $t$, and $\Sigma_{t}^{\kappa}$ is a composite of the variances of the parameter estimations $\Sigma^{\theta}$, as calculated in Equation 11, and using gradients from the current trial $W_{t}^{\kappa, \theta}$.

The choice probability estimates of the opponent $p^{o p, \kappa}$ for each possible opponent level $\kappa$ are now aggregated by a probability weighted average into a single choice probability estimate:

$$
P_{t}^{o p}=\sum_{\kappa} \lambda_{t}^{\kappa} P_{t}^{o p, \kappa}
$$

The opponent's probability of choosing 1 is now estimated and can be used in the decision process to produce the agent's own choice, as seen in section The Decision Process.

\subsection{Getting Started with tomsup}

One of the advantages of computational models of cognitive processes is that the implications of the model can be worked out by simulating the model's behavior in a variety of situations. tomsup in particular allows for testing the $\mathrm{k}$-ToM model as it plays a wide set of game-theoretical situations (e.g. Matching Pennies or Prisoner's Dilemma), in interaction with a variety of different agents (e.g. other k-ToM or less sophisticated agents), within different possible settings (e.g. repeated interactions with the same opponent, or round robin tournaments). In order to better understand the setup of the tomsup package, we start with the 
case of two simple agents interacting, followed by a simple example using k-ToM agents. Lastly, we will show how to run a simulation using multiple agents as well as how to plot the evolving beliefs of a k-ToM agent. The appendix contains tutorials for more complex uses of tomsup, like specifying $k$-ToM's starting beliefs, creating custom agents, and using tomsup agents for experimental stimuli.

In the simple scenario two agents are playing the Matching Pennies game against each other. One agent hides a penny in one hand: choosing 0 could indicate hiding it in the left hand, while choosing 1 indicates the right. The other agent has to guess where the penny is. If the second agent guesses correctly (chooses the same number as the first agent), it wins and the first agent loses. In other words, the second agent must match their decision while the first agent tries to avoid it. In this example, one of the agents implements the Random Bias strategy (e.g. has a 60 percent probability of choosing right over left), while the other implements a classic Q-learning strategy (a model free reinforcement learning mechanism updating the expected reward of choosing a specific option on a trial by trial basis).

The user first has to install the tomsup package developed using python 3.6 (Van Rossum \& Drake, 2009). The package can be downloaded and installed using pip:

pip3 install tomsup

The latest and less tested developmental build can also be installed directly from the github repository.

git clone https://github.com/KennethEnevoldsen/tomsup.git

cd tomsup

pip3 install -e .

Both approaches will also install the required dependencies. Now tomsup can be imported into Python following the lines;

import tomsup as ts

We will also set a arbitrary seed to ensure reproducibility;

import random

import numpy as np 
random.seed(1995) \# The year of birth of the first author

$\mathrm{np} \cdot \mathrm{random} \cdot \mathrm{seed}(1995)$

\# NumPy and Python uses two different random seeds.

First we need to set up the Matching Pennies game. As different games are defined by different payoff matrices, we set up the game by creating the appropriate payoff matrix using the PayoffMatrix class.

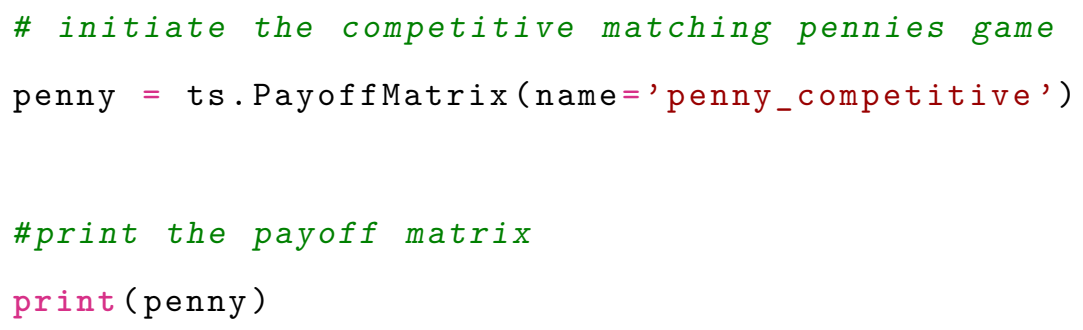

The payoff matrix of agent 1

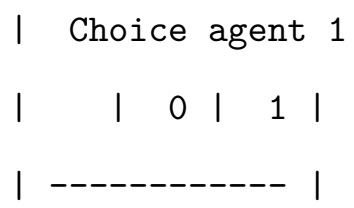

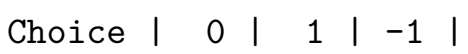

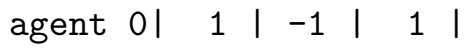

The Matching Pennies game is a zero sum game, meaning that for one agent to get a reward, the opponent has to lose. Agents have thus to predict their opponents' behavior, which is ideal for investigating ToM. Note that to explore other payoff matrices included in the package, or to learn how to specify a custom payoff matrix, the user can type the help(ts.PayoffMatrix) command. 
Then we create the first of the two competing agents:

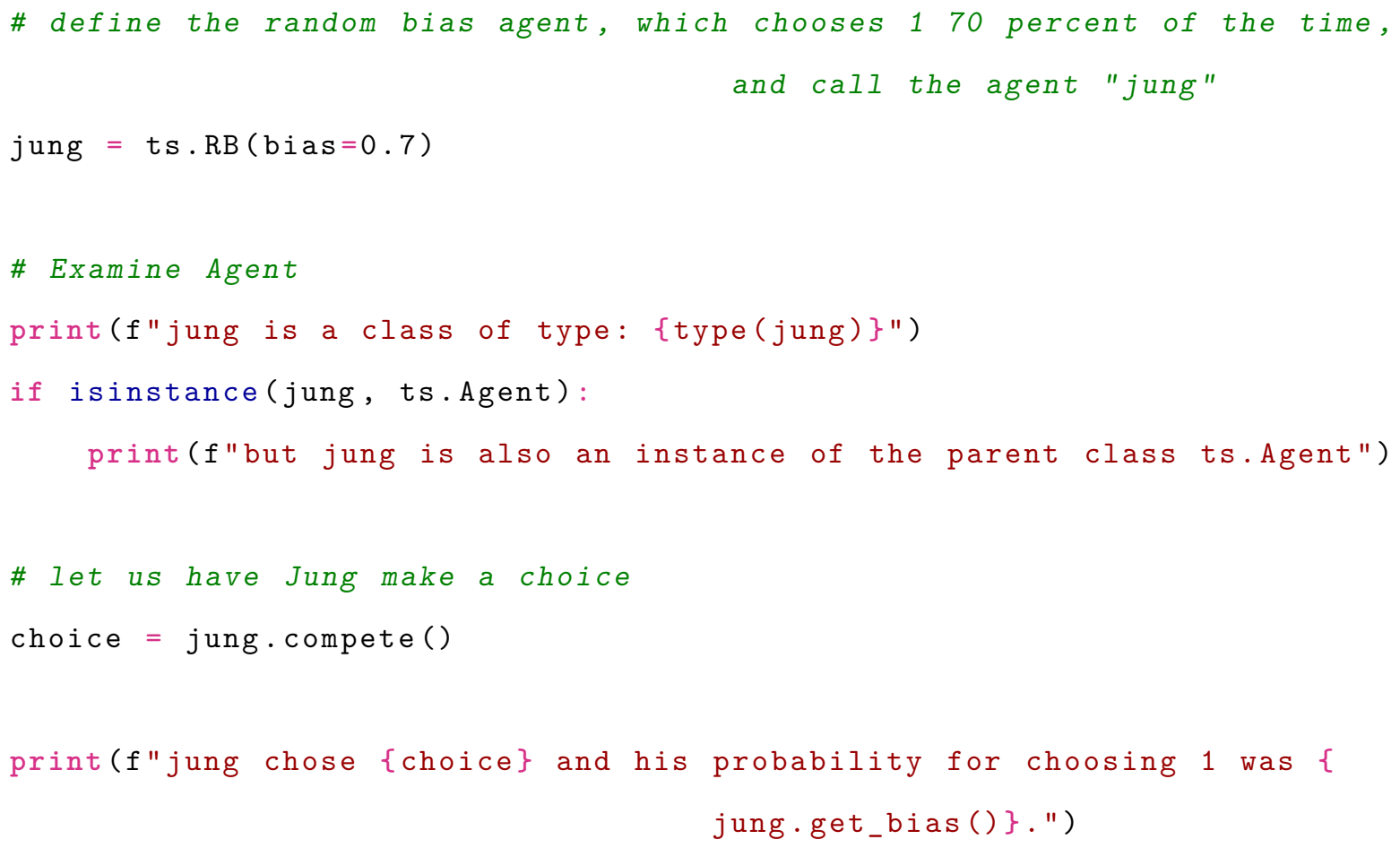

jung is a class of type: <class 'tomsup.agent.RB'>

but jung is also an instance of the parent class ts.Agent

jung chose 1 , and its probability for choosing 1 was 0.7 .

Note that it is possible to create one or more agents simultaneously using the convenient create_agents () and passing any starting parameters to it in the form of a dictionary.

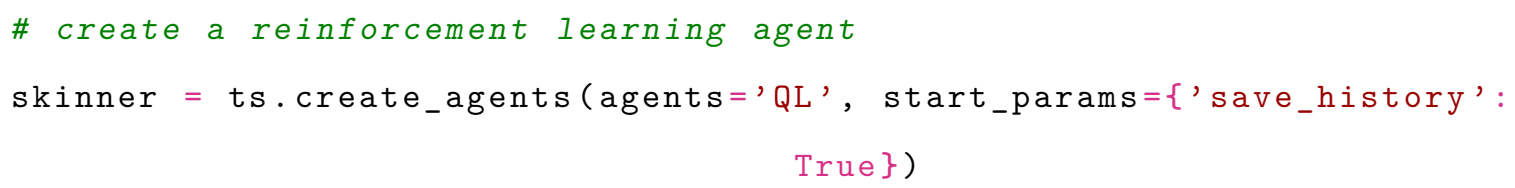

The full list of strategies currently implementable with tomsup can be found with the function valid_agents (). It is also possible to create custom agents, as per the tutorial found in Appendix B.

Now that both agents are created, we have them play against each other.

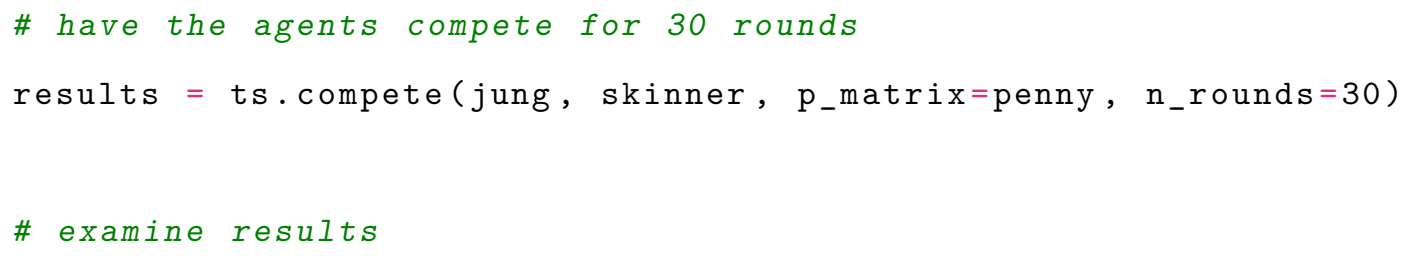


print(results.head()) \#inspect the first 5 rows of the dataframe

$\begin{array}{rrrrrr} & \text { round } & \text { choice_agent0 } & \text { choice_agent1 } & \text { payoff_agent0 } & \text { payoff_agent1 } \\ 0 & 0 & 1 & 1 & 1 & -1 \\ 1 & 1 & 1 & 0 & -1 & 1 \\ 2 & 2 & 1 & 0 & -1 & 1 \\ 3 & 3 & 1 & 0 & -1 & 1 \\ 4 & 4 & 0 & 0 & 1 & -1\end{array}$

The data frame stores the choice of each agent as well as their resulting payoff. Simply summing the payoff columns would determine the winner.

\section{Using $k$-ToM agents}

In the following we present a simple simulation study of two $k$-ToM agents playing the penny game against each other.

We will start off by creating a 0-ToM agent with default priors and save_history=True to examine the workings of it. The setting save_history is disabled by default to save on memory use, which is especially relevant for ToM agents with high sophistication levels. It is important to note that, for computational ease and for comparability with the implementation in the VBA toolbox (Daunizeau et al., 2014; Devaine et al., 2017), parameter values are inputted and saved on transformed scales. The behavioural temperature $\beta$ and the volatility $\sigma$ are both log-transformed, and the dilution $d$ is in log-odds. Use help (ts. TOM) for an overview.

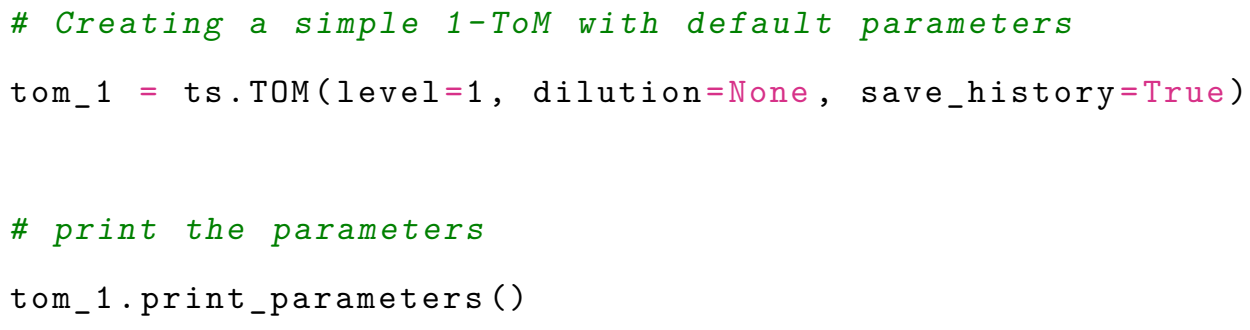


Note that k-ToM agents as default begin with agnostic starting beliefs. These can be seen in detail and specified as desired, as shown in Appendix A.

To increase the agent's tendency to choose 1 we could simply increase its bias. Similarly, if we want the agent to behave in a more deterministic fashion we can decrease the behavioural temperature. When the parameter values are set, we can play the agent against an opponent using the .compete () method, where agent denotes the agent in the payoff matrix ( 0 or 1$)$ and the op_choice denote the choice of the opponent during the previous round.

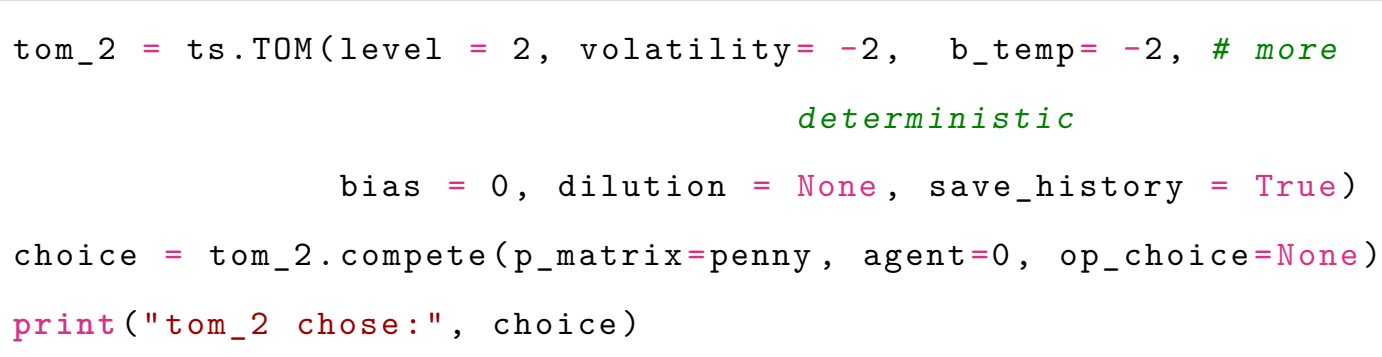

tom_2 chose: 1

The user is recommended to have the 1-ToM and the 2-ToM agents compete by using the ts. compete ( ) as it was done in the previous section. However, to make the process more transparent for the user, we here instead show the process in a simple for loop:

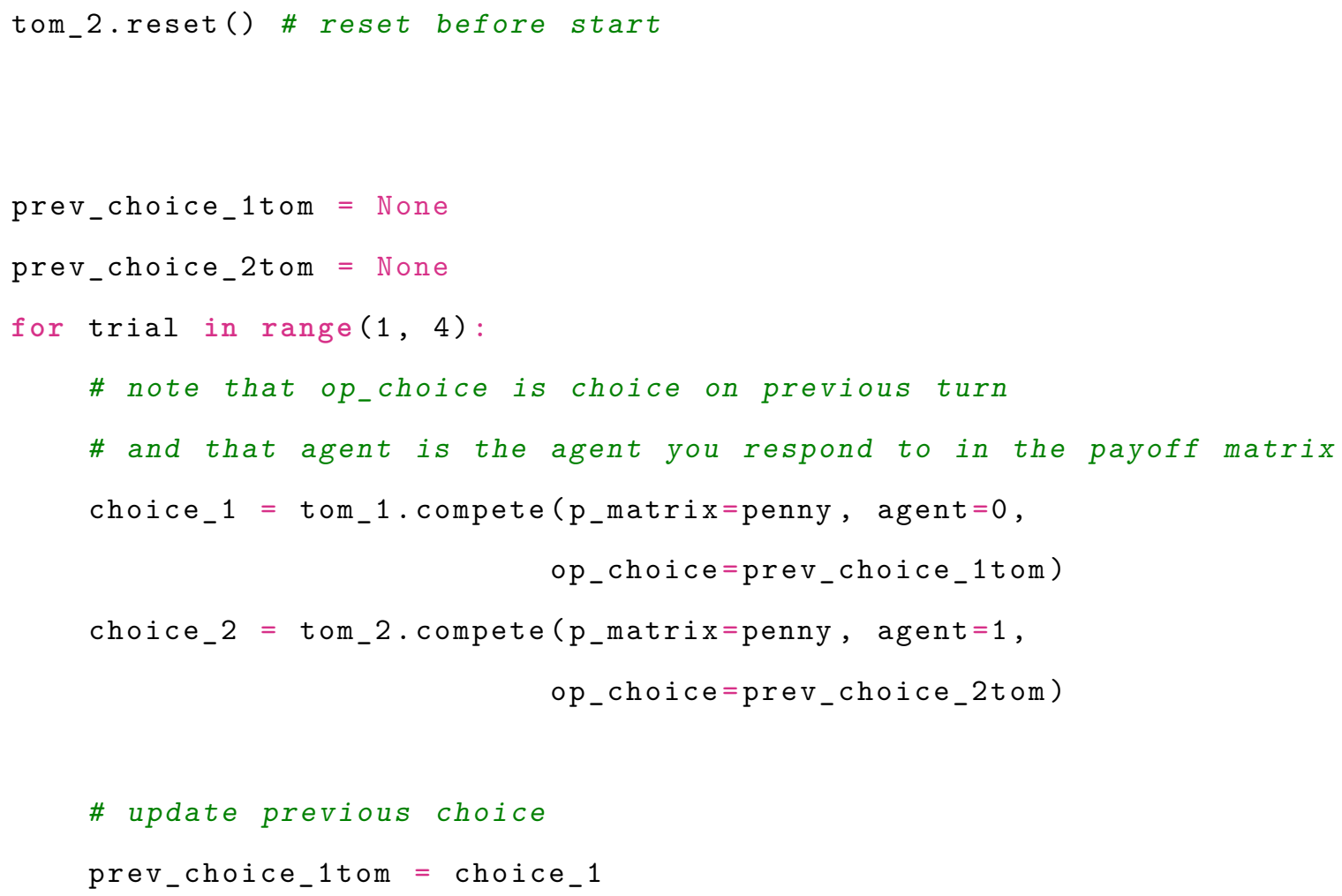




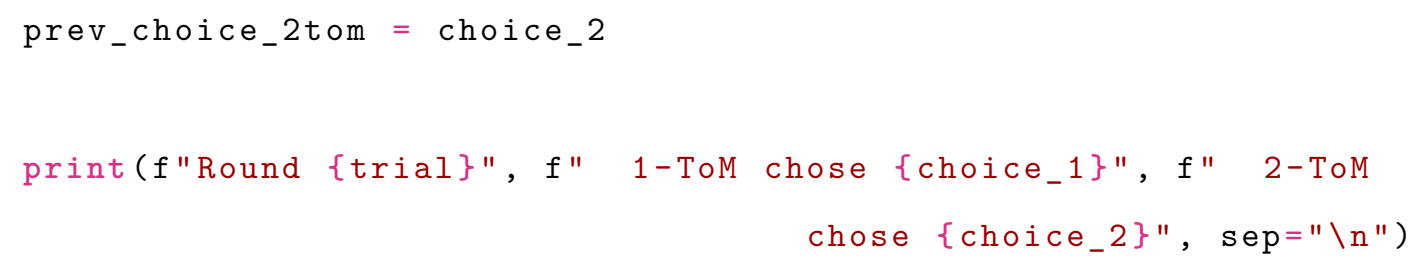

Round 1

$1-$ ToM chose 1

2-ToM chose 1

Round 2

1-ToM chose 1

2-ToM chose 0

Round 3

$1-$ ToM chose 1

2-ToM chose 1

Incidentally, using a for loop like this is also the easiest way of implementing tomsup agents as experimental stimuli. A tutorial for doing this with the software PsychoPy (Peirce et al., 2019) can be found in Appendix C.

tomsup also has convenient functions for exploring and visualizing the internal states of ToM agents:

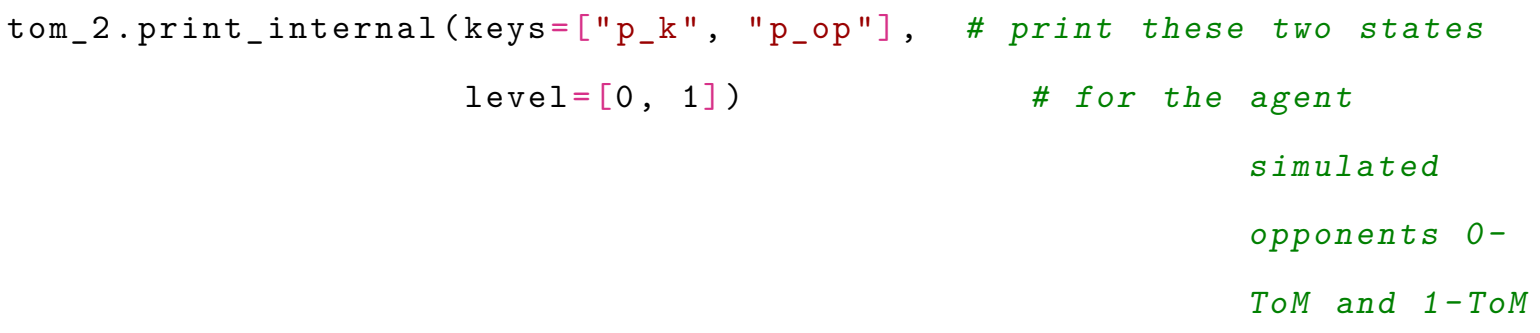

opponent_states

I $0-$ ToM

I | opponent_states

| $\quad$ own_states

I 1-ToM

| | opponent_states 


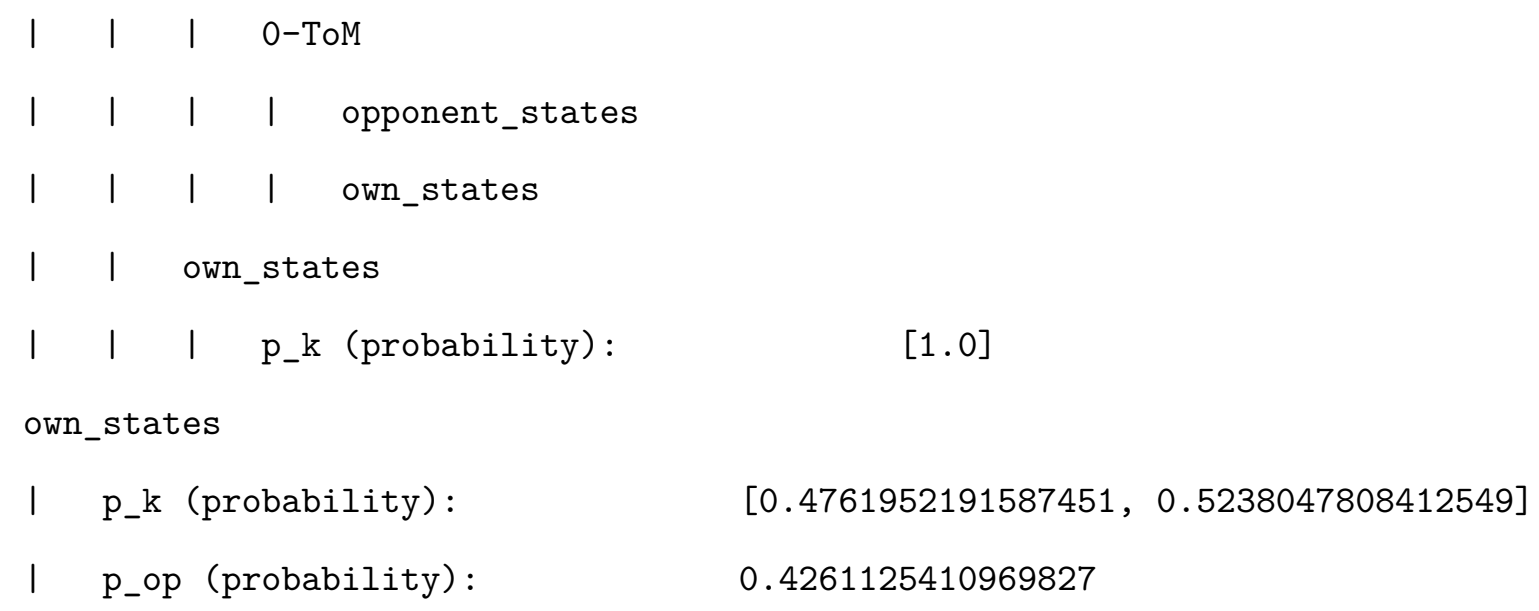

For instance, we can note that the estimate of the opponent's sophistication level (p_k) slightly favors a 1-ToM as opposed to a $0-\mathrm{ToM}$ and that the average probability of the opponent choosing one (p_op) slightly favors 1 (which was indeed the option the opponent chose). These estimates are quite uncertain due to the few rounds played. This function also displays information on which transformations the internal states are stored in. More information on each type of internal state can be found using help(ts.print_internal).

\section{Simulating Multiple Agents and Visualizing Results}

The above syntax is useful for small setups. However, the user might want to build larger simulations involving several agents to simulate data for experimental setup or test underlying assumptions. The package provides syntax for quickly iterating over multiple agents, rounds and even simulations. Note also that 'n_jobs' can be used to parallelize the computation, significantly speeding up the simulation (See Appendix D for details). We will here show a quick example along with how to visualize the results and internal states of ToM agents.

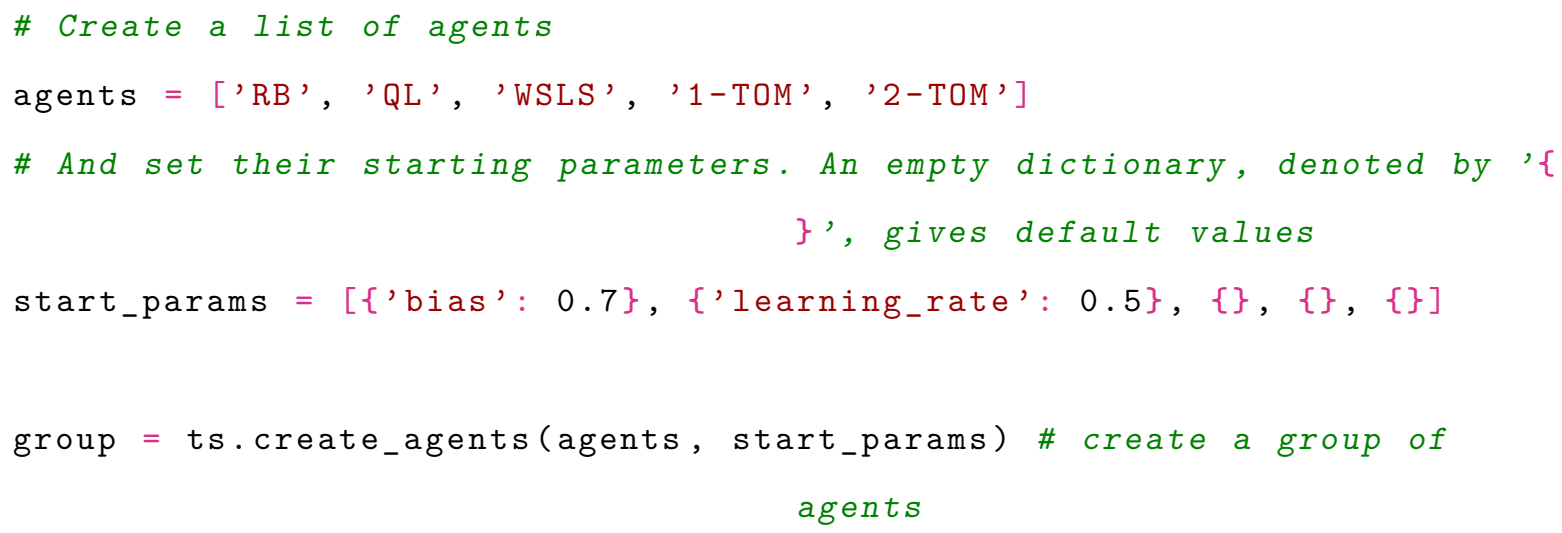


\# Specify the environment

\# round_robin e.g. each agent will play against all other agents

group.set_env(env='round_robin')

\# Finally, we make the group compete 20 simulations of 30 rounds

results $=$ group. compete(p_matrix=penny, n_rounds $=30$,

n_sim=20, save_history=True)

Currently the pair, ('RB', 'QL'), is competing for 20 simulations, each containing 30 rounds.

Running simulation 1 out of 20

Running simulation 2 out of 20

$[\ldots]$

Running simulation 20 out of 20

Simulation complete

Following the simulation, a data frame can be extracted as before, with additional columns reporting simulation number, competing agent pair (agent0 and agent1) and if save_history=True it will also add two columns denoting the internal states of each agent, e.g. estimates and expectations at each trial.

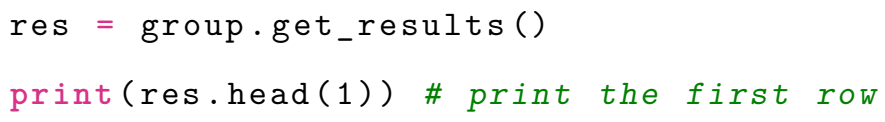


n_sim round choice_agent0 choice_agent1 payoff_agento payoff_agent1 \

0

0

0

1

$-1$

$\begin{array}{lll} & \text { history_agent0 } & \text { history_agent1 agent0 } \\ 0 \quad\{\text { 'choice': } 0\} & \{\text { 'choice': } 0, \text { 'expected_value0': } 0.5, \ldots\}\end{array}$

agent0 agent1

$0 \quad \mathrm{RB} \quad \mathrm{QL}$

The package also provides convenient functions for plotting the agent's choices and performance. The following code plots results in Figure 3 and 4.

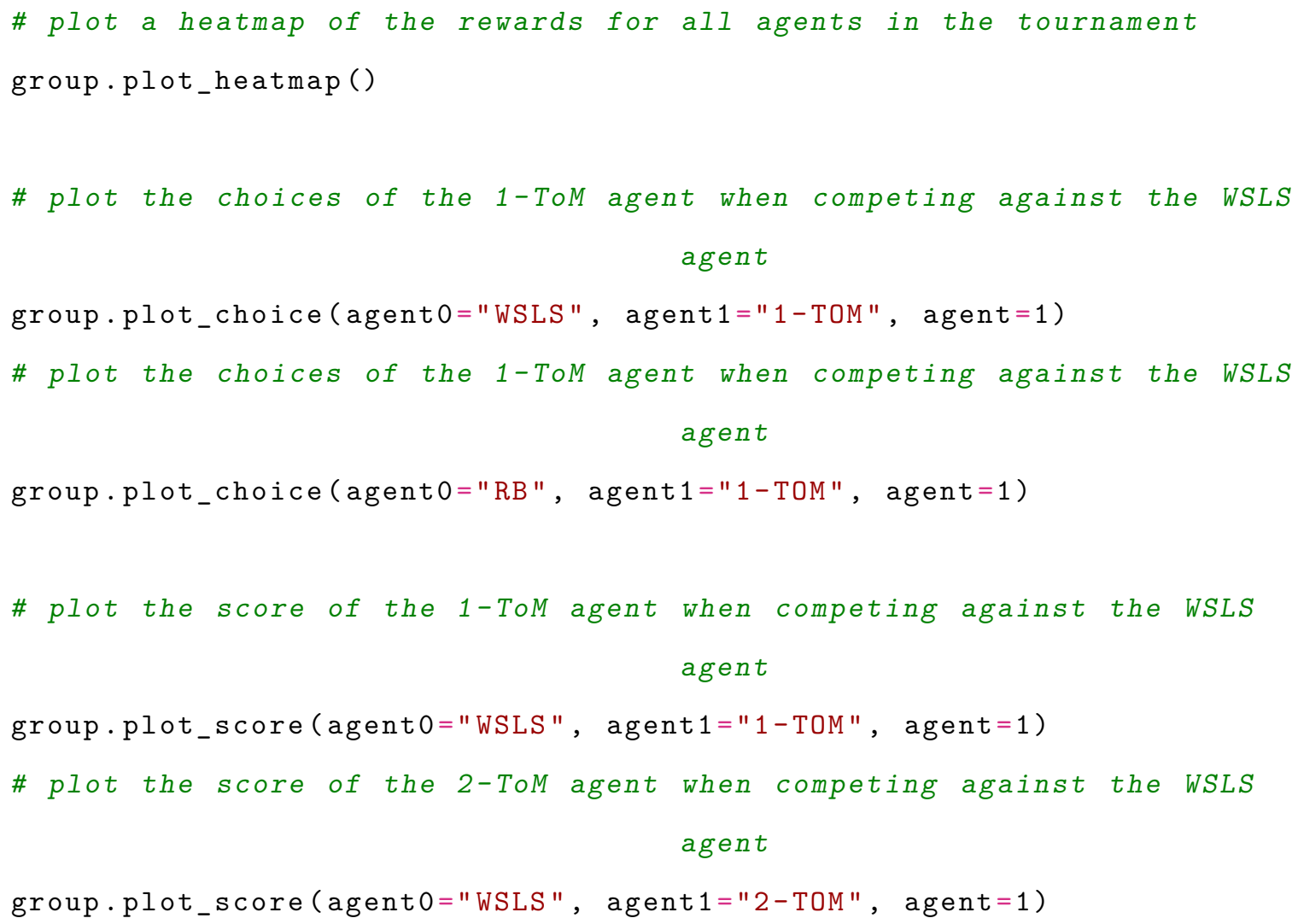




\section{Figure 3}

A heatmap displaying the average score across simulations for each competing pair the score denotes the score of the agent (x-axis) when playing against to the opponent (y-axis). The score in the parenthesis denotes the $95 \%$ confidence interval.

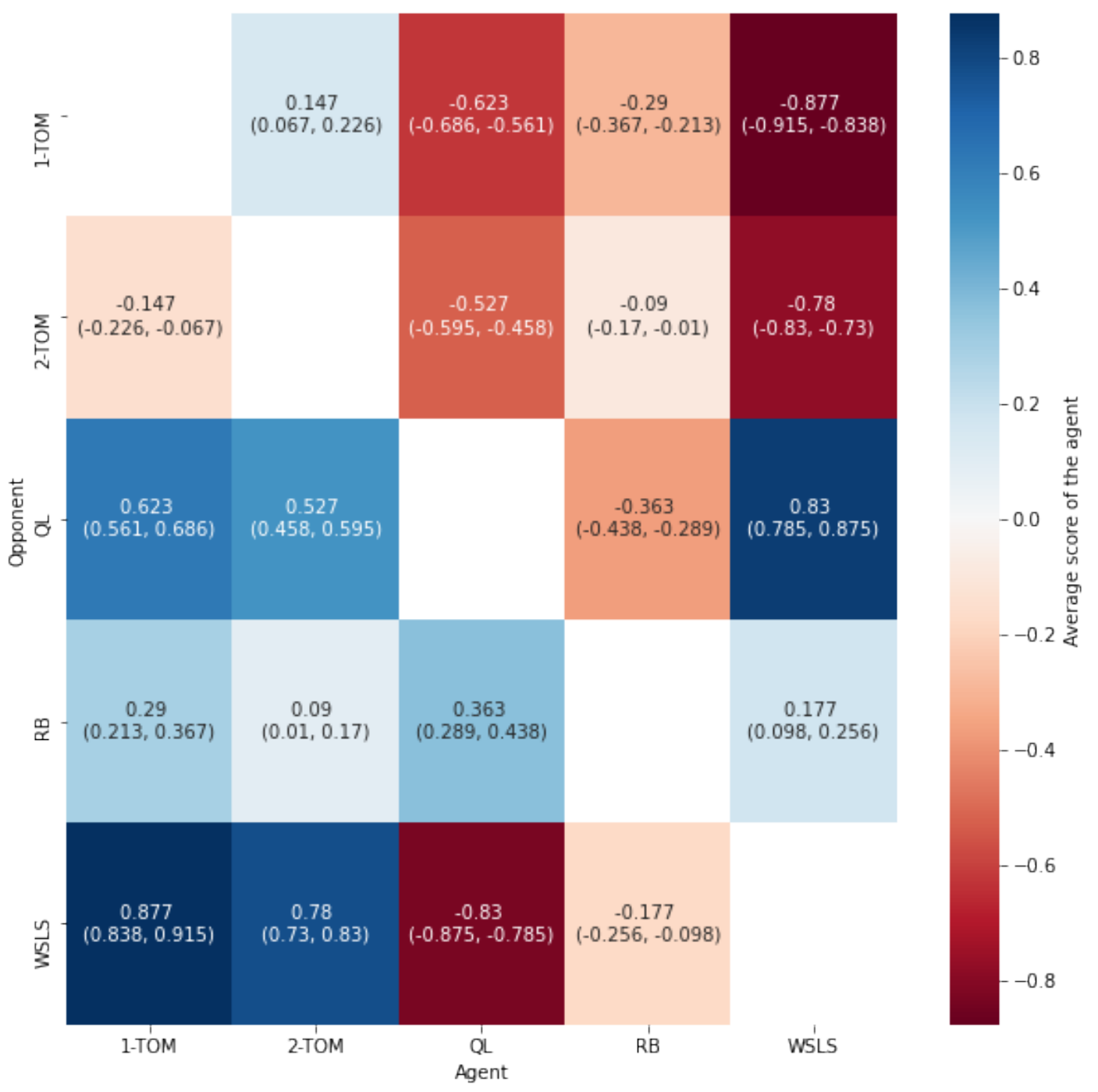

Figure 3 shows that k-ToM models compare favorably against simpler agents such as the QL. Furthermore, both 1-ToM and 2-ToM perform especially well against the WSLS agent. This is because the WSLS essentially implements heuristically a deterministic and high learning rate bias estimation strategy which can be approximated well as a low-volatility, low-temperature, high-dilution 0-ToM model. Similarly, a QL can be approximated using a low-temperature and low-volatility 0-ToM. We see that all agents are able to win against the RB agent, although with different levels of efficiency. Finally, we see that 1-ToM and 2-ToM are 
behaviourally similar in this task, and against most of the opponents, except when playing against each other, in which case 2-ToM slightly outperforms 1-ToM. Although note that we expect the 1 -ToM model playing against the 2 -ToM to become increasingly more difficult to predict: unable to capture the 2-ToM dynamics, the 1-ToM likely grows more uncertain and therefore behaviourally more random. In general, 2-ToM is less efficient in predicting the simpler opponents than 1-ToM, also presumably because the more complex model requires more observations in order to become certain of its opponent's behavioural patterns (i.e., they require more data to infer relatively precise parameter value distributions, having more parameters to fit). These patterns could be further investigated in different ways, but one would be to look at average choice and reward patterns over time, as exemplified in Figure $4 \mathrm{c}$ and $4 \mathrm{~d}$. Here we see clearly that both $1-\mathrm{ToM}$ and 2-ToM become almost perfectly able to predict the behaviour of the WSLS agent, with a slightly longer learning time for the 2-ToM agent. In Figure 4a and $4 \mathrm{~b}$ we also see how different opponents elicit different behavioural patterns in a 1-ToM agent: when such agent plays against an RB agent, it exhibits a bias estimation behaviour, while it enters an oscillating choice pattern against the WSLS. This is but a taste of how to visualize the simulations run through tomsup. One might investigate how these behavioural patterns vary depending on the game theoretic context, or investigate such things as how well agent's choices can be predicted from their or their opponents' earlier actions, across contexts, or summarize the behaviour in ways that rely less on averaging across simulations, which might hide important differences and nuances. The plotting functions included in tomsup are ultimately meant as convenient tools for initial investigation, while visualizations and analyses more appropriate for a specific research question can easily be made with the information contained in the results data frame produced by tomsup. 


\section{Figure 4}

Choice and score plotted over rounds. The blue/bold lines indicate the mean score or choice across simulations, with each simulation represented by a grey/thin line.

(a)

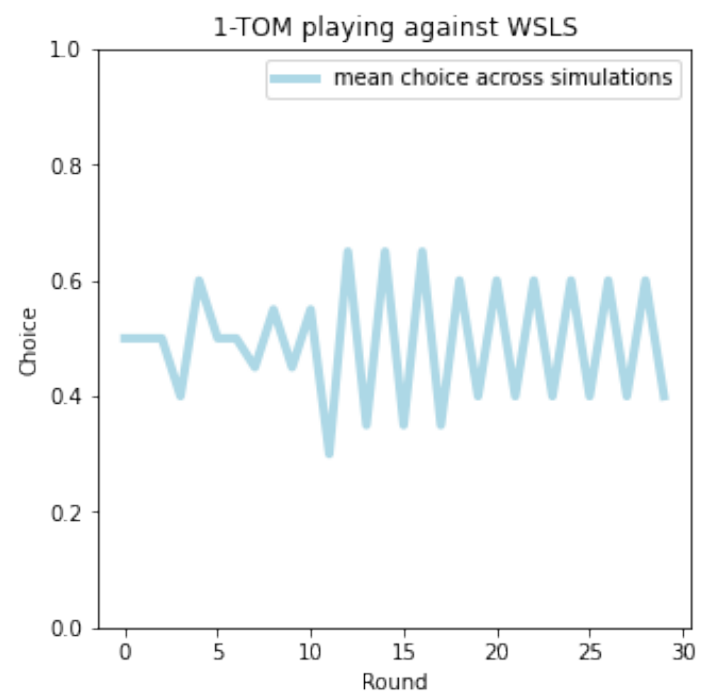

(c)

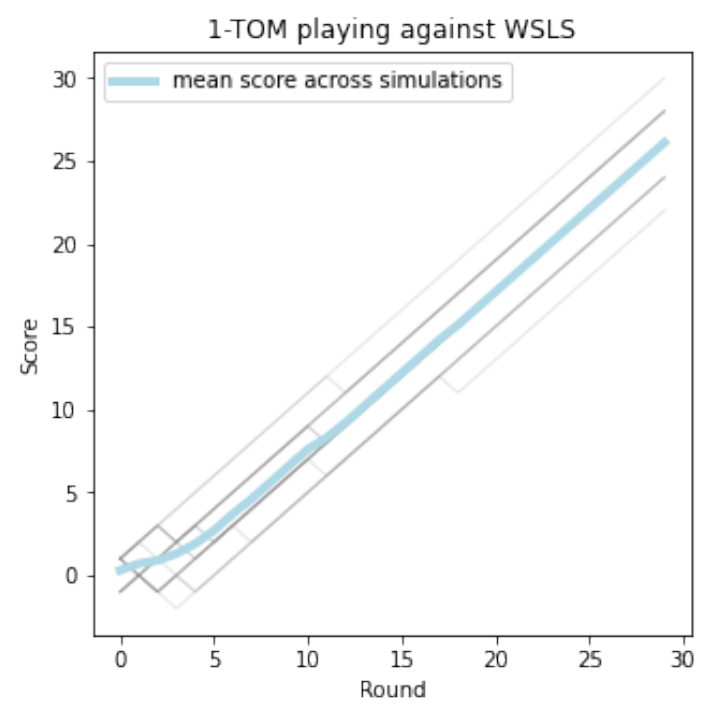

(b)

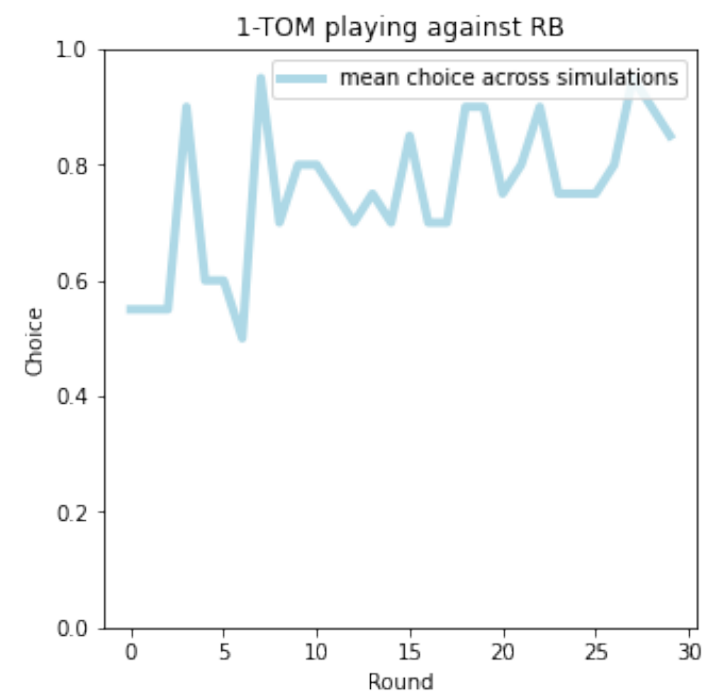

(d)

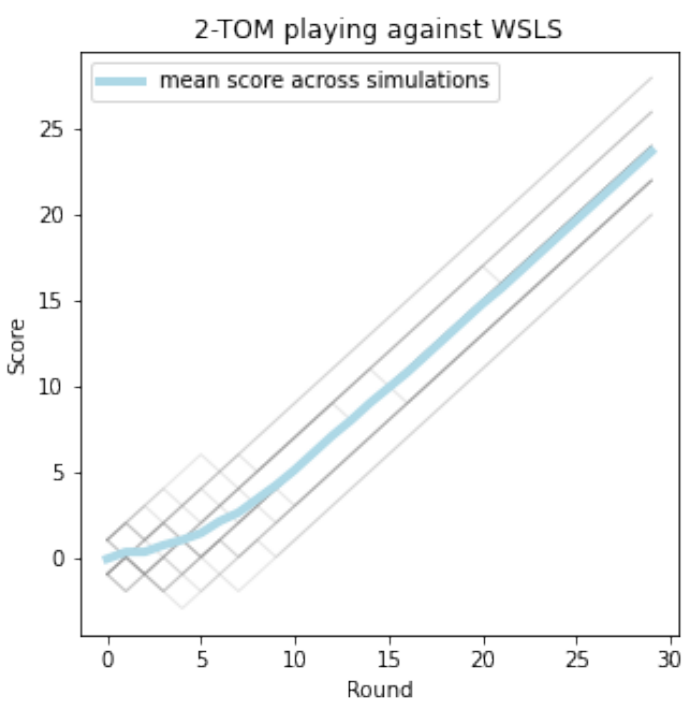

We note that besides the more generic plots applicable to all simulated agents, tomsup contains dedicated plotting shortcuts for $k$-ToM's internal states. This includes the estimate of the opponent's sophistication level, as seen in Figure 5, where it can be seen that the 2-ToM 
agent on average correctly estimates its 1-ToM opponent's sophistication level of $1 .^{2}$

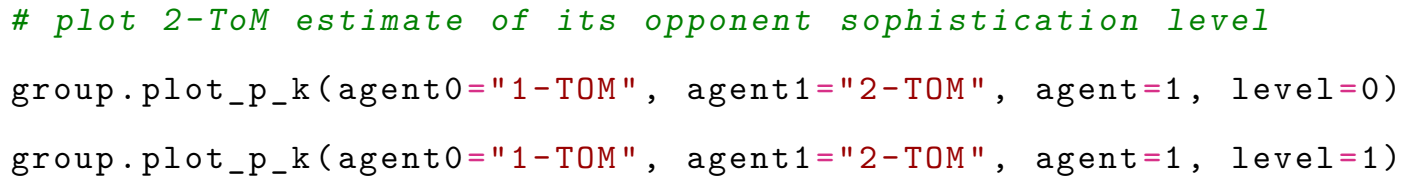

\section{Figure 5}

The 2-ToM agent's estimations for its opponent having a sophistication level of 0 (left) or 1 (right). In almost all simulations, the 2-ToM agent correctly estimates its opponent's level to be 1. The blue/bold lines indicate the mean score across simulations, with each simulation represented by a grey/thin line.
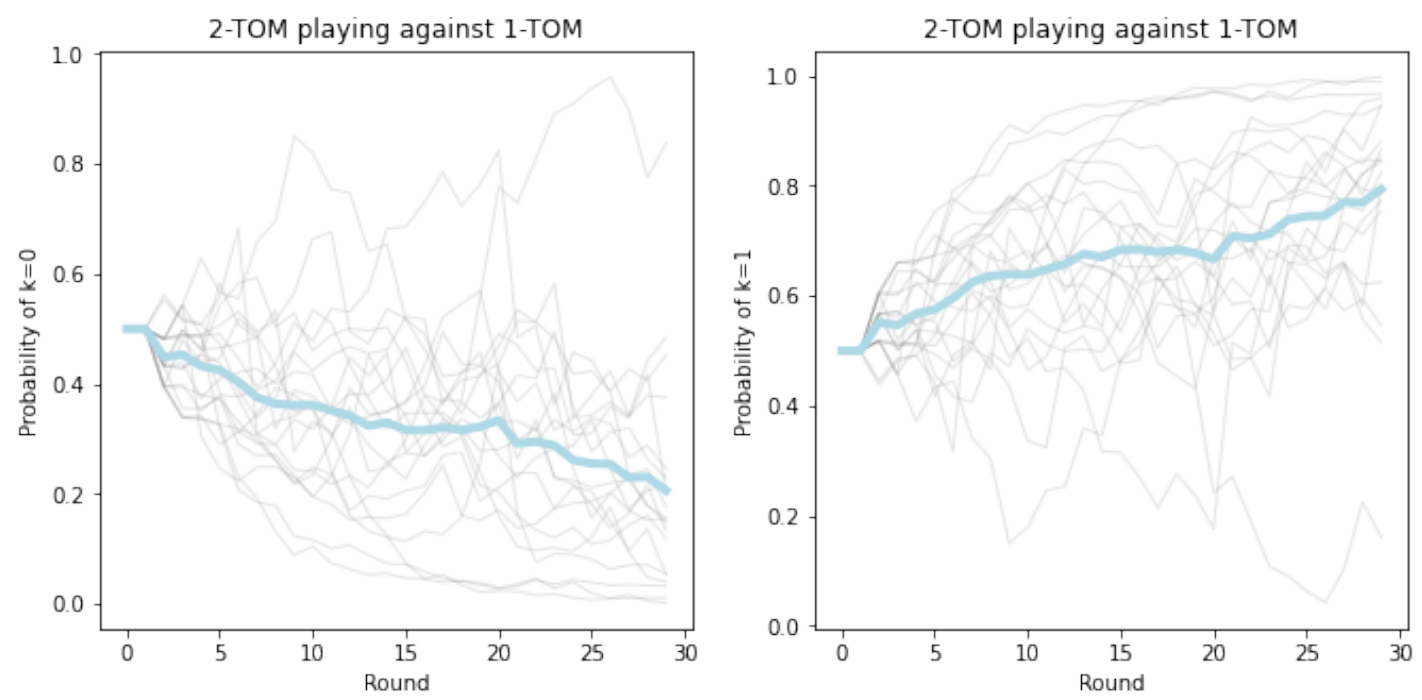

It is also easy to plot $k$-ToM's estimates of its opponent's model parameters. As an example, the following code plots 2-ToM's estimate of a 1-ToM opponent's volatility and bias (shown in Figure 6). In this example, the 2-ToM agent approaches a correct estimate of the opponent's (default) volatility of $-2(\log$ scale) as well as correctly estimates its opponent as having no inherent bias.

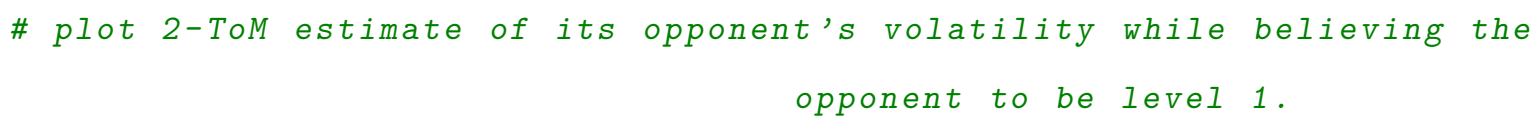

\footnotetext{
${ }^{2}$ Note that 2-ToM is slightly biased away from concluding an opponent to be at level 0 . This is probably because the higher-level 1-ToM model have more parameters and is more flexible, and therefore is easier to fit to the data. Future versions of the algorithm could include penalties for increased amounts of parameters.
} 


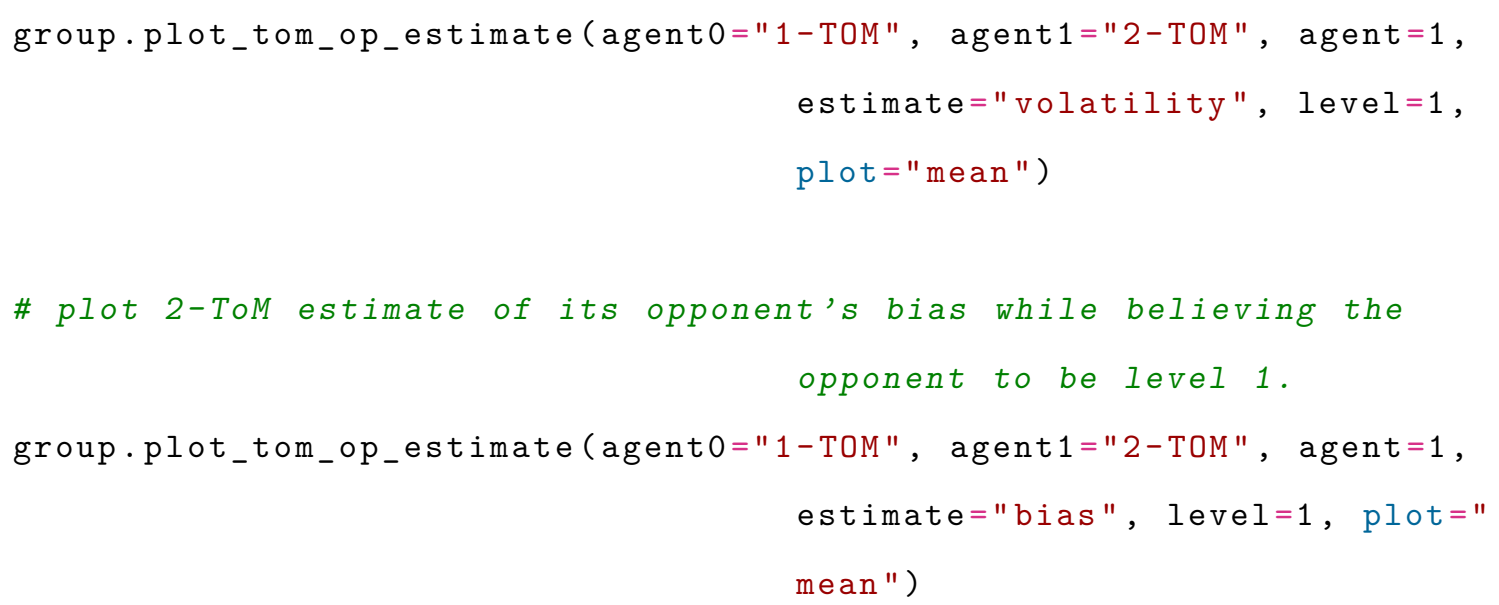

\section{Figure 6}

2-ToM's estimate of 1-ToM's volatility (left) and bias(right). With time, the 2-ToM agent approaches a correct estimate of its opponent having a volatility of -2 , and on average correctly estimates its opponent to have a bias of 0 . The blue/bold lines indicate the mean score across simulations, with each simulation represented by a grey/thin line.
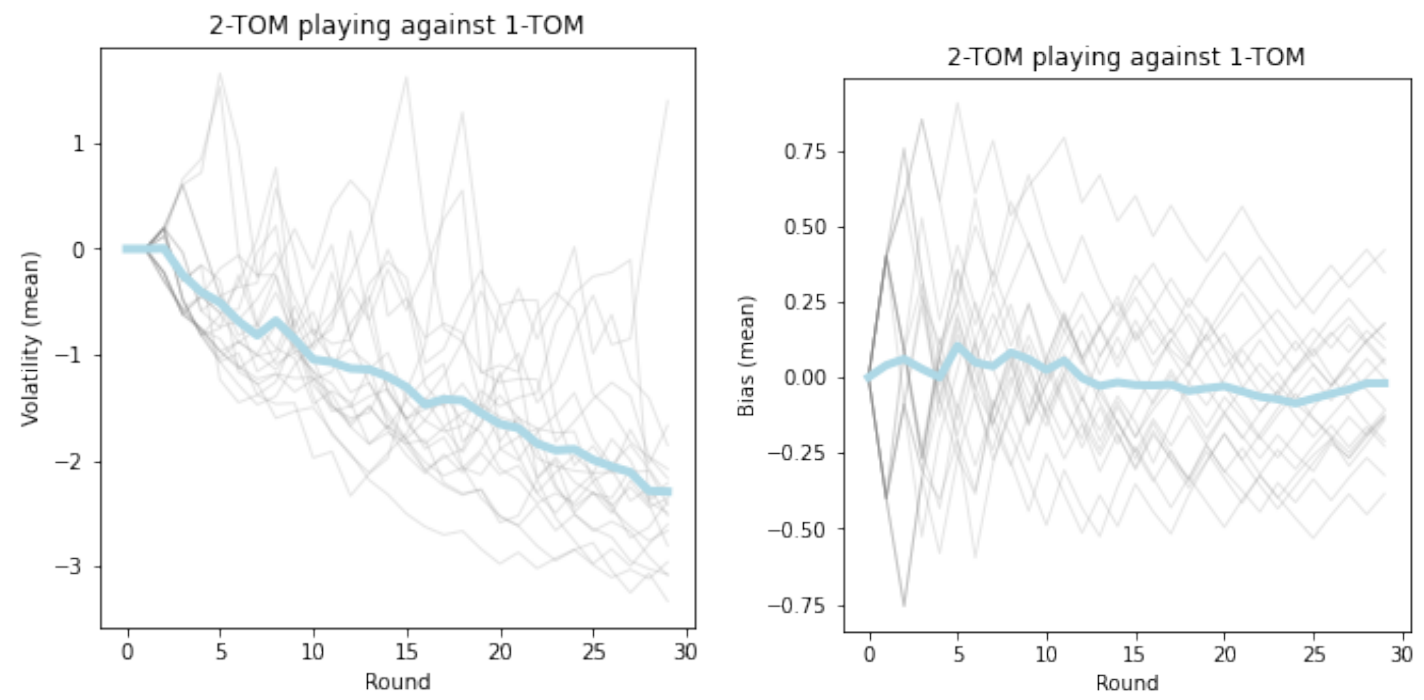

Use help(ts.AgentGroup.plot_tom_op_estimate) for information on how to plot the other estimated parameters or $k$-ToM's uncertainty in these parameters. The full list of changing variables of an agent for any given trial can be found in the history column in the results data frame, which contains all of $k$-ToM's internal states, including choice probability, gradient and parameter estimates and uncertainties for itself and all its recursively simulated possible opponents. 
Note that tomsup is being further developed, so updated documentation, examples and tutorials can be found on the Github repository for the tomsup package. ${ }^{3}$

\subsection{Online Parameter Recovery}

We assessed the ability of $k$-ToM models to correctly infer the properties of their opponents, e.g. their level of recursion $(k)$, bias, temperature and volatility. We ran a simulation experiment, where a 3-ToM model with default starting parameters (consistent with the VBA package to allow for comparison) competed against $1500 \mathrm{k}$-ToM agents with a wide range of different starting parameters: the full combinatorial grid of 3 sophistication levels $k \in\{0,1,2\}, 10$ values of volatility $\Sigma \in(-3,-1), 10$ values of bias, $b \in(-1,1)$, and 5 values of behavioural temperature $\beta \in(-1.5,-0.5)$. The agents were set to play the competitive matching pennies game over 100 rounds.

We see in Figure 7 that sophistication and bias were reasonably estimated within 10-30 rounds, similarly we see that 3-ToM obtains a reasonable differentiation in sophistication level after few rounds, improving consistently, even faster if the opponent is a 0-ToM. Temperature and volatility were harder to estimate, showing continuous improvement over 100 trials, although not the exact estimate. We see that the model is able to deal with incorrect initial conditions. The relatively poor estimation of volatility is due to a more basic non identifiability of the parameters: differences in behavior can be equally explainable by adjusting the estimated opponent's volatility or the estimated opponent's estimate of the agent's own volatility. Parameter recovery patterns are similar when 3-ToM makes estimates against ToM agents of all three sophistication levels. Recursive parameters such as the opponents estimation of the agent's sophistication level or bias could similarly be extracted, but we have chosen to focus primarily on the parameters which would typically be of interest. In addition to providing information about how well ToM agents can infer parameters about each other, it also serves as a preliminary parameter recovery test for when using an on-line 3-ToM agent to infer sophistication levels of participants based on experimentally observed behaviour, as we do in the next section. Note that a thorough parameter estimation based on

\footnotetext{
${ }^{3}$ https://github.com/KennethEnevoldsen/tomsup/tree/master/tutorials
} 
the k-ToM model, and also a full parameter and model recovery study, would benefit from being done using variational or sampling based Bayesian model inversion, approaches which among other possible advantages - would allow using all observations for estimating parameters on trials before the last, avoiding sub-optimal inference.

\section{Figure 7}

A 3-ToM's estimation of the internal states when playing against 0-ToM, 1-ToM or 2-ToM agent with a range of different starting parameters as specified by a grid. The fit denotes a linear regression for estimations except for the sophistication level which is the median. The shading denoting the $25 \%$ lower and $75 \%$ upper quantile interval.

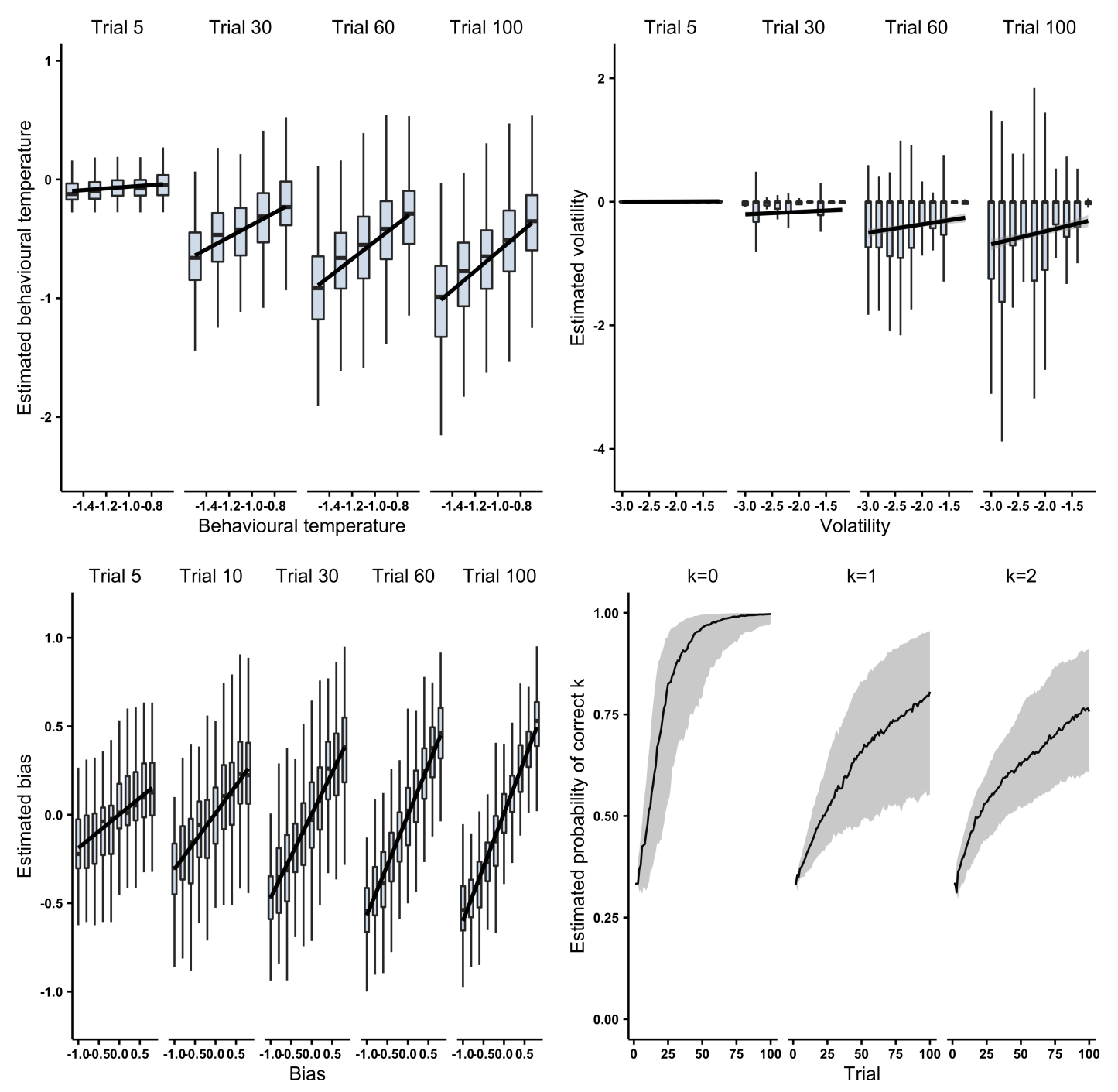




\subsection{An experimental use case}

To illustrate how tomsup can be concretely used in an experimental setting, we provide an example - loosely inspired by (D'Arc et al., 2018) - of how to design a study involving human participants and how to analyze the resulting data.

We set up to test the impact of framing the interaction with artificial agents as social (playing against another participant) or non-social (pulling the levers of a slot machine). Within each of these conditions, the participants had to play against agents at different levels of complexity (0-, 1- and 2-ToM). The participants played 20 trials against each agent in each condition, for a total of 120 trials. We implemented the paradigm by interfacing tomsup with PsychoPy, for details on the code implementation see Appendix $\mathrm{C}$ and the github repository of the project https://github.com/KennethEnevoldsen/tomsup/. 47 undergraduate students at Aarhus University (26 women and 21 men, mean age 20.3) participated in the study, which was approved by the local University Ethical Board and Data Protection Agency. In order to assess the impact of the experimental manipulations (framing and complexity of the agents) we showcase two complementary approaches: a more traditional analysis of differences in performance as a consequence of the conditions, and a more explicit cognitive modeling analysis of the strategies used by the participants as a function of the experimental conditions. A first analysis relying on this experimental setup is to identify whether the experimental conditions do indeed make a difference for average performance.

Figure 8 shows that players in general perform better when the opponent is framed as a slot machine as compared to being framed as another human player. This difference disappears, however, when the opponent is sufficiently complex, at which point players win as often as they lose. Given the high uncertainty around the mean estimates, we complement them with a plot displaying changes over time and a statistical analysis. 


\section{Figure 8}

Average payoffs across different sophistication levels of the simulated opponent, and whether the opponent was presented as a slot machine or a human. Error bars display the $95 \%$ confidence interval

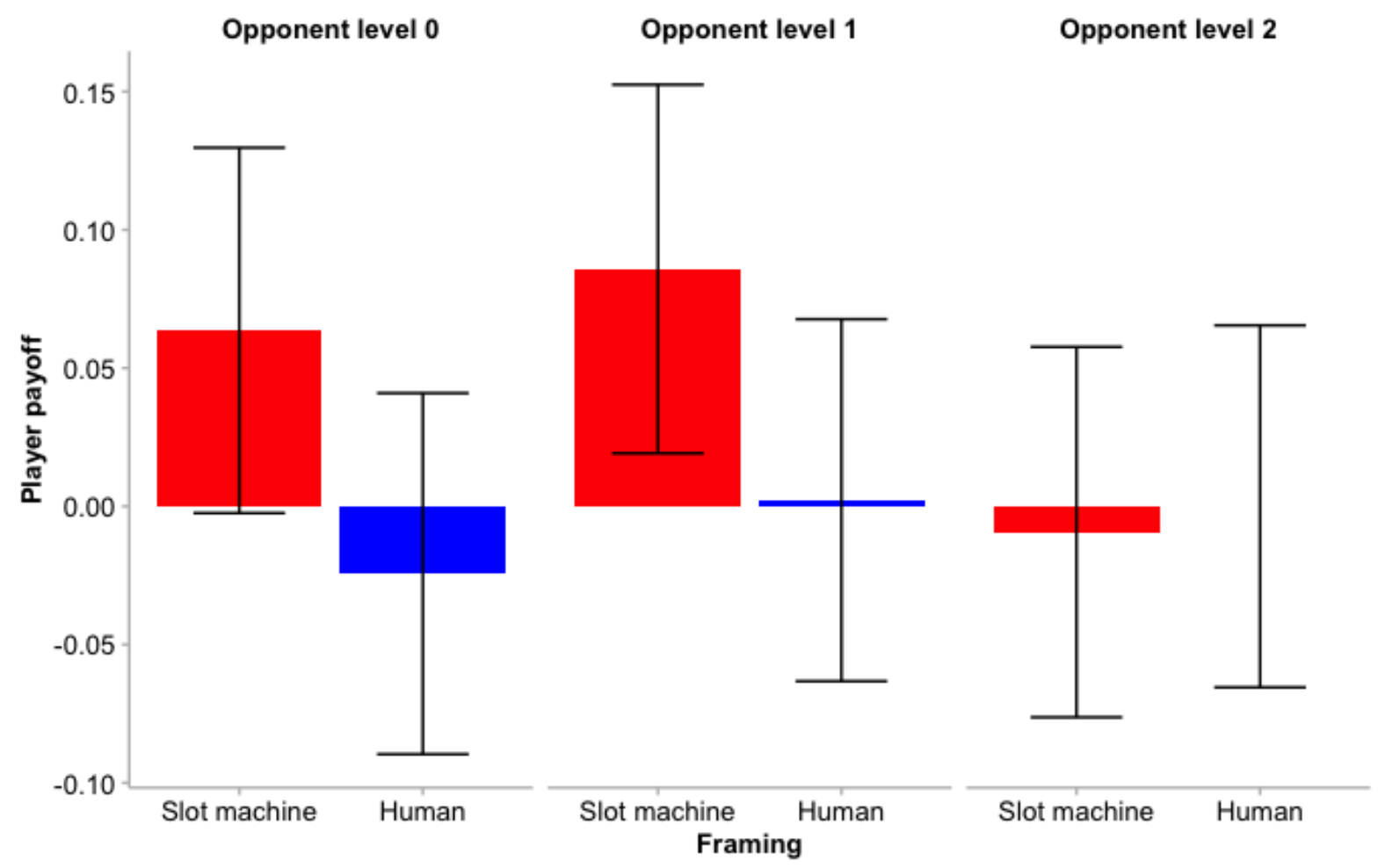

If we look at how performance develops over time within each experimental condition, figure 9 shows that the difference is due to the late trials. Participants learn only gradually to play effectively against the agents, and only against those framed as slot machines. This suggests that experimental setups should use more than 20 trials to allow learning to take place. 


\section{Figure 9}

Cumulative payoffs over trial time for different opponent sophistication levels, and whether the opponent was presented as a slot machine or a human. Shaded area shows the $95 \%$ confidence interval

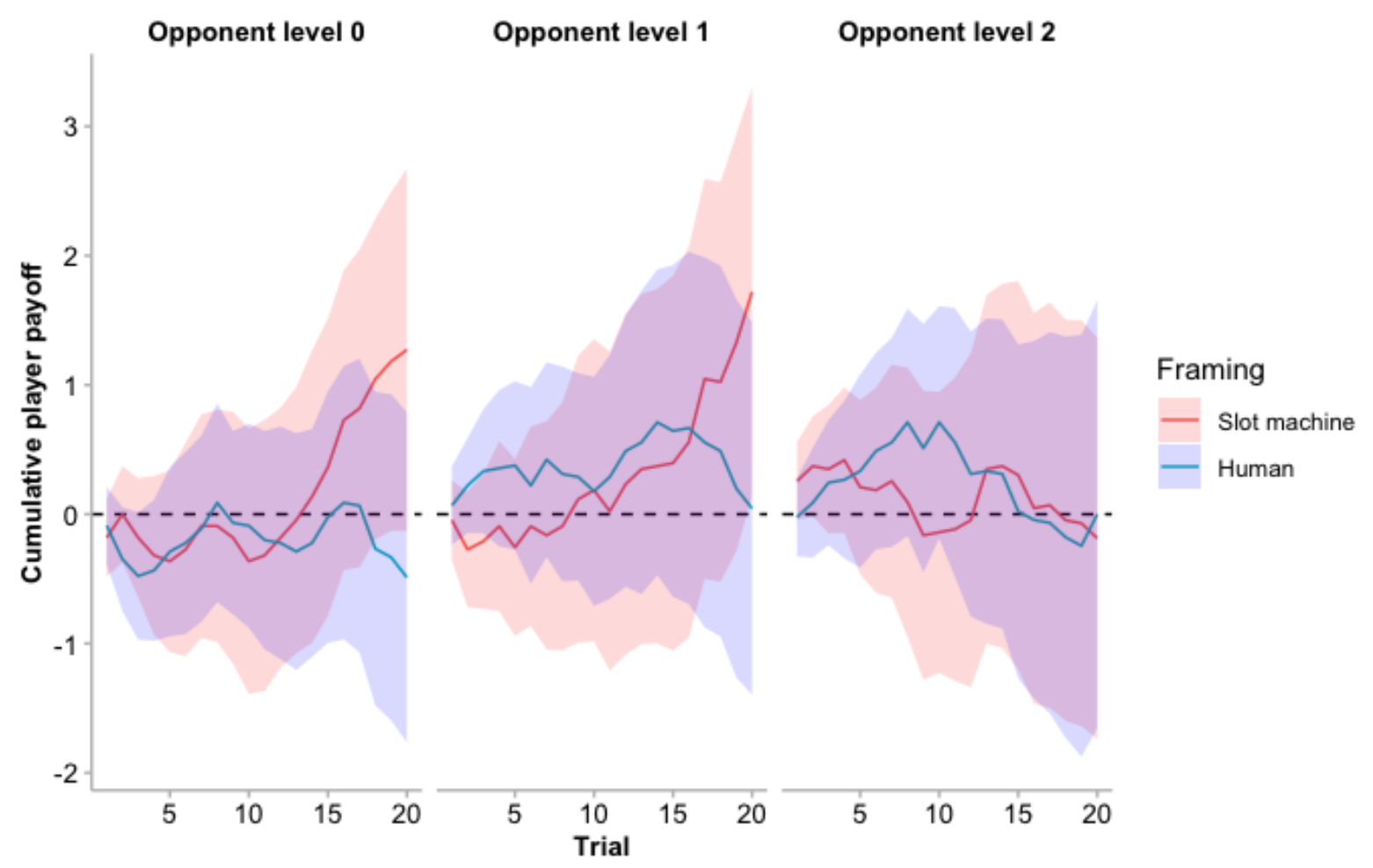

These observations are confirmed by a statistical analysis of the performance data (see https://github.com/KennethEnevoldsen/tomsup/, for full code of the implementation). We first build a Bayesian multilevel logistic regression, conditioning payoff on framing and opposing agent and all coefficients fully varying by participant. Performance in the non-social framing is indeed higher than the social framing: 3 percent increase in performance (95\% CIs: 00.05 , Evidence Ratio: 24.97 , credibility: $96 \%{ }^{4}$ ). Consistently with previous findings by d'Arc et al (2020), we also find that in the non-social condition performance decays as a function of complexity, while it stays constant in the social condition (interaction: $-0.05,95 \%$ CIs: -0.11 0.02, ER: 8.35; credibility: 89\%; non-social: -0.04 , 95\% CIs: -0.08 0.01, ER: 8.26, credibility:

\footnotetext{
${ }^{4}$ The evidence ratio is a Bayes factor between the hypothesis and its alternative computed via the Savage-Dickey density ratio method. Credibility refers to the percentage of samples (from the estimated distributions) compatible with the hypothesis.
} 
89\%; social: 0.01, 95\% CIs -0.03 0.06, ER: 2.16, credibility: 68\%). Analogously, we can see that performance increases over trials for participants in the non-social condition playing against 0-ToM (increase of 16 percent, 95\% CIs: 0.07 0.25, ER 221, credibility $=100 \%$ ) and 1-ToM (increase of 15 percent, 95\% CIs: 0.07 0.22, ER 665, credibility $100 \%$ ), but not in any other condition.

A second complementary analysis of the data can set a high level k-ToM agent to observe the participants' behavior and accordingly infer their level of sophistication in the different conditions. Here we use a 3-ToM agent.

Figure 10 shows that - at least as estimated by the model - participants tend to use a more sophisticated level of recursion (2-ToM) when playing against the simplest agent (0-ToM), across framing. However, participants also use a 2-ToM strategy when playing against a 1-ToM agent in the social framing. This latter pattern is not reflected in the performance analysis and constitutes one of the advantages of theory-driven cognitive modeling of the data, more concerned with mechanisms than with raw performance. 


\section{Figure 10}

An observing 3-ToM's inferences on the sophistication level of human players, depending on the framing and the sophistication level of the opponent. Shaded area shows the 95\% confidence interval

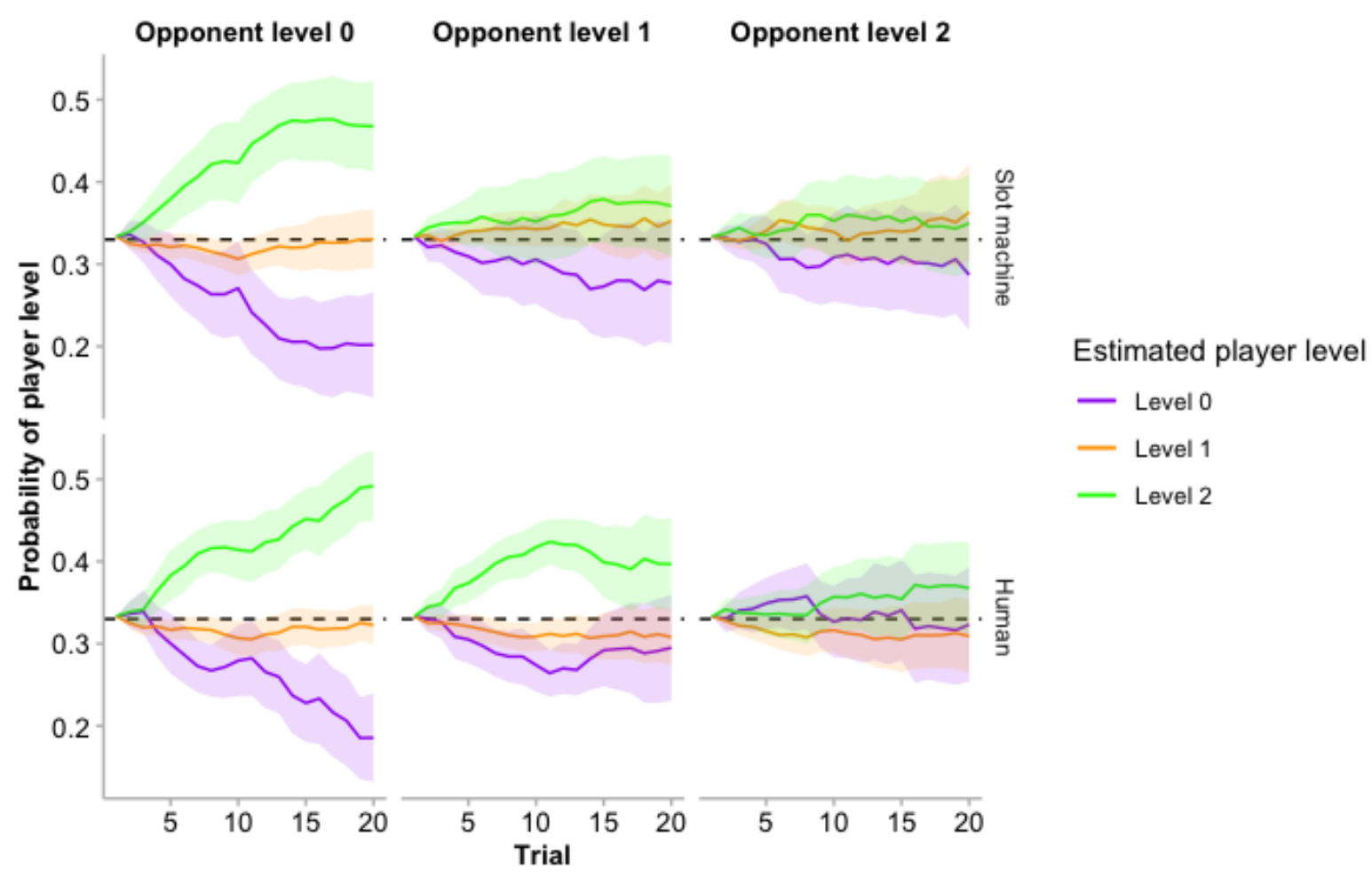

Importantly, it should be noted that these analyses are primarily intended as a demonstration of functionality and not a substantial experimental investigation.

\section{Discussion}

The current (1.0.0) version of tomsup provides a wide range of tools to better understand via simulations and experiments a recursive computational model of ToM ((Devaine et al., 2017)), as well as simpler non-recursive and heuristic models. Agents with a variety of computational strategies can be set against each other in a variety of game-theoretical situations, within diverse interactional and tournament settings. This allows for a deeper understanding of the implications of the different models and parameter values on performance and how they vary across contexts. For instance, one could assess how many levels of recursion can actually be inferred in these scenarios and in which conditions that would make a difference for 
performance, and compare that with empirical data. Thoroughly investigating in which games and against which opponents models behave differently or similarly to each other allows for assessments of model discriminability and facilitates experimental designs that best allows for inferring whether participants employ Theory of Mind. As a second crucial contribution, besides providing a tutorial on how to run these simulation studies, we also provide one on how to integrate computational agents and settings in experimental setups in real-time. This allows the assessment of human performance in similar settings as the simulations above, for easier comparison. We provide examples of data analysis and visualization for both simulation and experimental studies, as well as the code to implement them, to facilitate intuitive use of the package. This can for example be used to assess effects of framing on the use of recursive ToM, as well as whether these minimal 2-choice scenarios are indeed challenging enough or rich enough in terms of information and possible strategies for participants to actually engage in recursive thinking. Finally, we showcase how the package can be used to validate the models via parameter recovery, assessing whether the agents are able to infer the correct parameters. These functionalities can also be used to analyze empirical data, de facto fitting $\mathrm{k}$-tom models to the data and inferring parameter values.

While the package already provides great value for investigating Theory of Mind and (at least one of) its computational implementations, there are several limitations and needs for future development. In particular, in the following we discuss: 1) the need to develop and implement additional and more nuanced models of theory of mind;2) the need to develop more realistic and perhaps challenging interactional settings,; and 3) the need to complement the current setup with easy tools to fit and compare models on participants' data.

Additional models of theory of mind. tomsup is currently focused on variational recursive ToM computational models, as detailed in Devaine et al ((Devaine et al., 2017)). We aimed at providing an intuitive and yet rigorous introduction to these highly promising models, and how to compare them with less sophisticated models. However, as Rusch et al., 2020 nicely illustrate there is a wealth of alternative models that could also be implemented (e.g., the influence model by Hampton et al. (2008)), and of additional dimensions that could enrich our current $\mathrm{k}-\mathrm{ToM}$ models. The most important limitation is that the model is dominantly reactive. 
$\mathrm{k}$-ToM models plan their choice to maximize their immediate chances of payoff, but lack long term adaptive behavior. In other words, k-ToM models do not strategically plan their choices to e.g. optimize their estimation of the opponents' strategies, nor to optimize future payoff (e.g. willingly deceiving the opponent). Lacking this, $k$-ToM agents cannot successfully play games like the Prisoner's Dilemma. $k$-ToM agents will tend to choose the dominant strategy of defecting, which has the strongest incentive at any specific trial no matter what the opponent will do, despite being sub-optimal in the long run. To overcome this limitation, the model would have to simulate a number of trials ahead given both possible current decisions. Similar strategies are increasingly discussed for computational models in other cognitive domains (e.g. active inference models (Friston et al., 2017)). A second dimension for future developments is the range of strategies that a k-ToM agent assumes are possible for an opponent. Current k-ToM agents assume that their opponent can only be either a Random Bias (0-ToM) or a k-ToM agent. However, other strategies are documented for human behaviors in game-theoretical settings, for instance, heuristics such as Tit-For-Tat (following the opponent's previous choice) or Win-Stay-Lose-Shift (keeping the same choice til it loses), (Nowak \& Sigmund, 1993). A more nuanced model of Theory of Mind could implement the possibility to represent minds (or at least strategies) different from one's own. Finally, the specific mathematical structure of $k$-ToM could be changed: it would be possible to include a prospect-theory based utility transformation of rewards, to use a choice kernel to account for tendencies to persevere, systematically explore, or to account for more "emotional" patterns, for instance jealousy, by explicitly considering the opponent's performance and including tendencies to punish opponents that have high scores (Kahneman \& Tversky, 2013; Ligneul, 2019; Steingroever et al., 2013).

Additional interactional settings. In terms of interactional settings, an obvious development would be to allow the creation of more complex structures, including letting agents interact in a network structure, or making the environmental structure change as a function of interactions. This could be done by integrating tomsup with packages like NetworkX for Python (Hagberg et al., 2008). More radically, one needs to consider the nature of the interactions and their settings. tomsup implements one-step binary choice games. However, it 
has been repeatedly argued (Doshi et al., 2014; Hula et al., 2018; Rusch et al., 2020) that advanced ToM is maximally useful, and therefore more likely to be employed in richer settings, with several steps and interactions involved in any given choice, multiple agents and changing environmental conditions. Therefore, more complex decision scenarios might be necessary to better explore the realistic use of ToM. This would of course require generalizing $\mathrm{k}$-ToM models to these situations where the payoff matrix i.e. the preferences of itself and the opponent are unknown and must be estimated, or to tasks with more than two options.

Model fitting and comparison for participant data. The package offers the possibility to integrate $\mathrm{k}-\mathrm{ToM}$ agents in experimental setups, testing actual participants. This allows the user to better evaluate how participants adapt to agents, implementing different kinds of cognitive strategies and Theory of Mind mechanisms. Participant's performance against ToM agents can be used as an indirect measure of their ToM abilities, and the $k$-ToM agents' inferences about those players might also be explored. However, these procedures only give very indirect access to information about participants' mental processes, and cannot be comparatively extended to more naturalistic settings where participants interact with other participants.This calls for fitting computational models of ToM directly to empirical data. Accordingly, we show in the use cases how to use the agents' inferences to estimate participants' parameters. However, more flexible computational cognitive modeling could be implemented, for example, in PyMC3 (Salvatier et al., 2016), STAN (Stan Development Team, 2018), as in the HBayesDM package (Ahn et al., 2017), or JAGS (Plummer, 2004), as in (Lee \& Wagenmakers, 2014).

Implementing the $\mathrm{k}$-ToM model in such a language would provide better diagnostic tools for assessing the model quality (Yao et al., 2018), as well as posterior estimation of the participants' parameters fully including uncertainty. Posterior distributions of parameter estimates would afford more reliable modeling of how ToM measures could co-vary with other behavioural, demographic or even neurological measures (Haines et al., 2020). This would also allow for empirically motivating distributions of $k$-ToM's parameters and priors that can be used in subsequent simulations. Finally, this implementation would provide better tools for model comparison, testing the current ToM model against other possible 
implementations, in order to make mechanistic inference about ToM in humans and other creatures capable of playing simple game theoretic games.

\section{Conclusion}

The tomsup package provides an accessible computational tool to simulate Theory of Mind based agents in game theoretic contexts. This allows for making simulation studies, investigating the implications of the models included in tomsup or custom self-specified models, and also makes it possible to make participants interact with simulated agents in experimental contexts. A tutorial on how to use tomsup and an example of a simulation study has been shown in this paper, along with an in-depth explanation of the computational k-ToM model implemented in the package. This hopefully allows researchers to ask questions about the mechanisms behind Theory of Mind in more robust ways based on exactly defined computational models, contributing to shared research on Theory of Mind, an important theoretical concept that is otherwise difficult to investigate empirically.

\section{Declarations}

\section{Acknowledgments}

AS was supported by a postdoctoral fellowship from the Carlsberg Foundation. PW, KE, and RF ideated the project. PW and KE contributed equally to the generation of the python package, example scripts, package tutorials, supervised by RF. AV, RF, and AS designed and implemented the data collection, and analyzed the data. PW and KE co-wrote the first draft of the manuscript; AS, AV, and RF critically contributed to the successive versions.

\section{Conflict of Interest}

The authors have no conflicts of interest to declare that are relevant to the content of this article.

\section{Open Practices Statement}

The materials used for and referenced in this article such as example script and package tutorial is available at https://github.com/KennethEnevoldsen/tomsup. Since the package is 
liable to be updated, a time-stamped, immutable version of the package as it was when the example simulations in this paper were made can be found on https://github.com/KennethEnevoldsen/tomsup/tree/Immutable_Publication_Branch. The data used for example analysis, which was made using that script, is stored on a Open Science Framework repository https://osf.io/p36bd/. 


\section{References}

Ahn, W.-Y., Haines, N., \& Zhang, L. (2017). Revealing neurocomputational mechanisms of reinforcement learning and decision-making with the hBayesDM package. Computational Psychiatry, 1, 24-57.

Apperly, I. A. (2012). What is "theory of mind"? Concepts, cognitive processes and individual differences. Quarterly Journal of Experimental Psychology, 65(5), 825-839.

Axelrod, R., \& Hamilton, W. D. (1981). The evolution of cooperation. Science (New York, N.Y.), 211(4489), 1390-6. https://doi.org/10.1126/SCIENCE.7466396

Baker, C., Saxe, R., \& Tenenbaum, J. (2011). Bayesian theory of mind: Modeling joint belief-desire attribution. Proceedings of the annual meeting of the cognitive science society, 33.

Baron-Cohen, S. (2000). Theory of mind and autism: A review, In International review of research in mental retardation. Elsevier.

Baron-Cohen, S., Leslie, A. M., \& Frith, U. (1985). Does the autistic child have a "theory of mind" ? Cognition, 21(1), 37-46. https://doi.org/10.1016/0010-0277(85)90022-8

Berecz, H., Tényi, T., \& Herold, R. (2016). Theory of mind in depressive disorders: A review of the literature. Psychopathology, 49(3), 125-134.

Bloom, P., \& German, T. P. (2000). Two reasons to abandon the false belief task as a test of theory of mind. Cognition, 77(1), B25-B31. https://doi.org/10.1016/S0010-0277(00)00096-2

Bosacki, S., \& Wilde Astington, J. (1999). Theory of mind in preadolescence: Relations between social understanding and social competence. Social development, 8(2), $237-255$.

Boucher, J. (2012). Putting theory of mind in its place: Psychological explanations of the socio-emotional-communicative impairments in autistic spectrum disorder. Autism: The International Journal of Research and Practice, 16(3), pmid 22297199, 226-246. https://doi.org/10.1177/1362361311430403

Brüne, M. (2005). "Theory of mind" in schizophrenia: A review of the literature. Schizophrenia Bulletin, 31(1), 21-42. 
Camerer, C. F. (2010). Behavioural game theory, In Behavioural and Experimental Economics. Springer.

Camerer, C. F., Ho, T.-H., \& Chong, J.-K. (2004). A cognitive hierarchy model of games. The Quarterly Journal of Economics, 119(3), 861-898.

Conway, J. R., Catmur, C., \& Bird, G. (2019). Understanding individual differences in theory of mind via representation of minds, not mental states. Psychonomic Bulletin \& Review, 26(3), 798-812. https://doi.org/10.3758/s13423-018-1559-x

Dale, R., Fusaroli, R., Duran, N. D., \& Richardson, D. C. (2013). The Self-Organization of Human Interaction, In Psychology of Learning and Motivation. Elsevier. https://doi.org/10.1016/B978-0-12-407187-2.00002-2

D’Arc, B. F., Marie, D., \& Daunizeau, J. (2018). A reverse Turing-test for predicting social deficits in people with Autism. bioRxiv, 414540. https://doi.org/10.1101/414540

Daunizeau, J., Adam, V., \& Rigoux, L. (2014). VBA: A probabilistic treatment of nonlinear models for neurobiological and behavioural data (A. Prlic, Ed.). PLoS Computational Biology, 10(1), e1003441. https://doi.org/10.1371/journal.pcbi.1003441

De Bruin, L., Van Elk, M., \& Newen, A. (2012). Reconceptualizing second-person interaction. Frontiers in Human Neuroscience, 6. https://doi.org/10.3389/fnhum.2012.00151

DeCanio, S. J., \& Fremstad, A. (2013). Game theory and climate diplomacy. Ecological Economics, 85, 177-187. https://doi.org/10.1016/j.ecolecon.2011.04.016

Dennett, D. C. (1978). Beliefs about beliefs. Behavioral and Brain sciences, 1(4), 568-570.

Deschrijver, E., \& Palmer, C. (2020). Reframing social cognition: Relational versus representational mentalizing. Psychological Bulletin.

Devaine, M., Hollard, G., \& Daunizeau, J. (2014a). The social bayesian brain: Does mentalizing make a difference when we learn? (J. Beck, Ed.). PLoS Computational Biology, 10(12), e1003992. https://doi.org/10.1371/journal.pcbi.1003992

Devaine, M., Hollard, G., \& Daunizeau, J. (2014b). Theory of mind: Did evolution fool us? (T. Zalla, Ed.). PLoS ONE, 9(2), e87619. https://doi.org/10.1371/journal.pone.0087619 Devaine, M., San-Galli, A., Trapanese, C., Bardino, G., Hano, C., Saint Jalme, M., Bouret, S., Masi, S., \& Daunizeau, J. (2017). Reading wild minds: A computational assay of 
Theory of Mind sophistication across seven primate species (J. O’Reilly, Ed.). PLoS Computational Biology, 13(11), e1005833.

https://doi.org/10.1371/journal.pcbi.1005833

Devezer, B., Nardin, L. G., Baumgaertner, B., \& Buzbas, E. O. (2019). Scientific discovery in a model-centric framework: Reproducibility, innovation, and epistemic diversity. PLOS ONE, 14(5), e0216125. https://doi.org/10.1371/journal.pone.0216125

Doshi, P., Qu, X., \& Goodie, A. (2014). Chapter 8 - decision-theoretic planning in multiagent settings with application to behavioral modeling (G. Sukthankar, C. Geib, H. H. Bui, D. V. Pynadath, \& R. P. Goldman, Eds.). In G. Sukthankar, C. Geib, H. H. Bui, D. V. Pynadath, \& R. P. Goldman (Eds.), Plan, activity, and intent recognition. Boston, Morgan Kaufmann.

https://doi.org/https://doi.org/10.1016/B978-0-12-398532-3.00008-7

Friston, K., FitzGerald, T., Rigoli, F., Schwartenbeck, P., \& Pezzulo, G. (2017). Active inference: A process theory. Neural Computation. https://doi.org/10.1162/NECO\\{\\\\}a\\{\\\\}00912

Guest, O., \& Martin, A. (2020). How computational modeling can force theory building in psychological science. https://doi.org/10.31234/osf.io/rybh9

Hagberg, A. A., Schult, D. A., \& Swart, P. J. (2008). Exploring Network Structure, Dynamics, and Function using NetworkX (G. Varoquaux, T. Vaught, \& J. Millman, Eds.). In G. Varoquaux, T. Vaught, \& J. Millman (Eds.), Proceedings of the 7th Python in Science Conference, Pasadena, CA USA.

Haines, N., Kvam, P. D., Irving, L. H., Smith, C., Beauchaine, T. P., Pitt, M. A., Ahn, W.-Y., \& Turner, B. (2020). Learning from the Reliability Paradox: How Theoretically Informed Generative Models Can Advance the Social, Behavioral, and Brain Sciences.

Hampton, A. N., Bossaerts, P., \& O’Doherty, J. P. (2008). Neural correlates of mentalizing-related computations during strategic interactions in humans. Proceedings of the National Academy of Sciences, 105(18), 6741-6746. 
Hula, A., Vilares, I., Lohrenz, T., Dayan, P., \& Montague, P. R. (2018). A model of risk and mental state shifts during social interaction. PLoS computational biology, 14(2), e1005935.

Kahneman, D., \& Tversky, A. (2013). Prospect theory: An analysis of decision under risk, In Handbook of the fundamentals of financial decision making: Part $i$. World Scientific.

Kampis, D., Karman, P., Csibra, G., Southgate, V., \& Hernik, M. (2020). A two-lab direct replication attempt of Southgate, Senju, \& Csibra (2007).

Lee, M. D., \& Wagenmakers, E.-J. (2014). Bayesian cognitive modeling: A practical course. Cambridge university press.

Ligneul, R. (2019). Sequential exploration in the iowa gambling task: Validation of a new computational model in a large dataset of young and old healthy participants. PLoS computational biology, 15(6), e1006989.

Morrison, K. E., DeBrabander, K. M., Jones, D. R., Ackerman, R. A., \& Sasson, N. J. (2020). Social Cognition, Social Skill, and Social Motivation Minimally Predict Social Interaction Outcomes for Autistic and Non-Autistic Adults. Frontiers in Psychology, 11. https://doi.org/10.3389/fpsyg.2020.591100

Nowak, M., \& Sigmund, K. (1993). A strategy of win-stay, lose-shift that outperforms tit-for-tat in the Prisoner's Dilemma game. Nature, 364(6432), 56-58. https://doi.org/10.1038/364056a0

Peirce, J., Gray, J. R., Simpson, S., MacAskill, M., Höchenberger, R., Sogo, H., Kastman, E., \& Lindeløv, J. K. (2019). PsychoPy2: Experiments in behavior made easy. Behavior Research Methods, 51(1), 195-203. https://doi.org/10.3758/s13428-018-01193-y

Pinkham, A. E., Morrison, K. E., Penn, D. L., Harvey, P. D., Kelsven, S., Ludwig, K., \& Sasson, N. J. (2020). Comprehensive comparison of social cognitive performance in autism spectrum disorder and schizophrenia. Psychological Medicine, 50(15), 2557-2565. https://doi.org/10.1017/S0033291719002708

Plummer, M. (2004). JAGS: Just another Gibbs sampler.

Premack, D., \& Woodruff, G. (1978). Does the chimpanzee have a theory of mind? Behavioral and Brain Sciences, 1(04), 515. https://doi.org/10.1017/S0140525X00076512 
Quesque, F., \& Rossetti, Y. (2020). What do theory-of-mind tasks actually measure? Theory and practice. Perspectives on Psychological Science, 15(2), 384-396. https://doi.org/10.1177/1745691619896607

Rusch, T., Steixner-Kumar, S., Doshi, P., Spezio, M., \& Gläscher, J. (2020). Theory of mind and decision science: Towards a typology of tasks and computational models. Neuropsychologia, 146, 107488. https://doi.org/10.1016/j.neuropsychologia.2020.107488

Salvatier, J., Wiecki, T. V., \& Fonnesbeck, C. (2016). Probabilistic programming in python using pymc3. PeerJ Computer Science, 2, e55.

Sasson, N. J., Morrison, K. E., Kelsven, S., \& Pinkham, A. E. (2020). Social cognition as a predictor of functional and social skills in autistic adults without intellectual disability. Autism Research, 13(2), 259-270. https://doi.org/10.1002/aur.2195

Schaafsma, S. M., Pfaff, D. W., Spunt, R. P., \& Adolphs, R. (2015). Deconstructing and reconstructing theory of mind. Trends in Cognitive Sciences, 19(2), 65-72. https://doi.org/10.1016/j.tics.2014.11.007

Schelling, T. (1978). Micromotives and macrobehavior. WW Norton \& Company, New York, Smaldino, P. (2020). How to translate a verbal theory into a formal model.

Stan Development Team. (2018). PyStan: The python interface to stan. http://mc-stan.org Steingroever, H., Wetzels, R., \& Wagenmakers, E.-J. (2013). Validating the PVL-Delta model for the Iowa gambling task. Frontiers in Psychology, 4, 898. https://doi.org/10.3389/fpsyg.2013.00898

Tylen, K., Allen, M., Hunter, B. K., \& Roepstorff, A. (2012). Interaction vs. observation: Distinctive modes of social cognition in human brain and behavior? A combined fMRI and eye-tracking study. Frontiers in Human Neuroscience, 6. https://doi.org/10.3389/fnhum.2012.00331

van Rooij, I., \& Baggio, G. (2020). Theory development requires an epistemological sea change.

Van Rossum, G., \& Drake, F. (2009). Python 3 reference manual. CreateSpace. 
Vélez, N., \& Gweon, H. (2020). Learning from other minds: An optimistic critique of reinforcement learning models of social learning. https://doi.org/10.31234/OSF.IO/Q4BXR

Watkins, C. J., \& Dayan, P. (1992). Q-learning. Machine learning, 8(3-4), 279-292.

Watson, A. C., Nixon, C. L., Wilson, A., \& Capage, L. (1999). Social interaction skills and theory of mind in young children. Developmental Psychology, 35(2), 386-391. https://doi.org/10.1037//0012-1649.35.2.386

Wimmer, H., \& Perner, J. (1983). Beliefs about beliefs: Representation and constraining function of wrong beliefs in young children's understanding of deception. Cognition, 13(1), 103-128. https://doi.org/10.1016/0010-0277(83)90004-5

Yao, Y., Vehtari, A., Simpson, D., \& Gelman, A. (2018, July 3). Yes, but Did It Work?: Evaluating Variational Inference, In International Conference on Machine Learning. International Conference on Machine Learning, PMLR. Retrieved September 26, 2021, from https://proceedings.mlr.press/v80/yao18a.html

Yoshida, W., Dolan, R. J., \& Friston, K. J. (2008). Game theory of mind. PLoS computational biology, 4(12).

\section{Glossary}

ToM Theory of Mind.

tomsup Theory of Mind Simulation using Python.

\section{Appendix A: Setting Starting Beliefs in the k-ToM Model}

The simulated ToM agents in the tomsup package use agnostic beliefs about their opponent's level probabilities $\lambda$ and choice probabilities $\mu$, while parameter estimation means $\mu^{\theta}$ are set to 1 . All variances $\Sigma^{\theta}$ and $\Sigma$ for parameter and choice probability estimation, respectively, are also set to 1 . Gradients for all parameters are 0 on the first trial, which means that no parameter estimates and variances are updated during the first trial. The tomsup package uses the same parameter values as the ones used in the VBA toolbox (Daunizeau et al., 2014) to enable comparison, but other settings are possible. 
One possibility is to set initial states directly in ts.TOM() using the priors. However, identifying the the correct input format might be prone to error, we therefore recommend to initialize the model with the defaults and then change the internal states to match the desired belief:

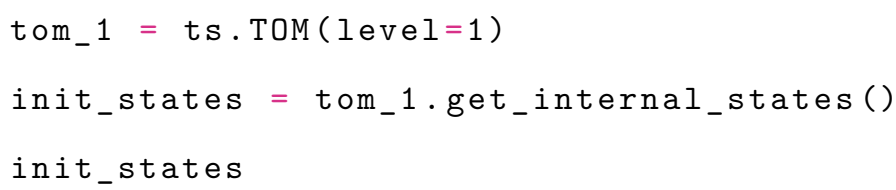

For instance, one might wish to change the initial assumption of the opponent's sophistication level, especially when the agent plays against human participants. This is defined in the variable priors ["own_states"] ["p_k"] which - by default - is set to $[0.5,0.5]$, i.e. agnostic about whether the opponent will use one level of or no recursion. Humans - we might assume - are more likely to use 1 level of recursion as opposed to 0 (no mentalizing of the agent). We can therefore change the agent's expectations as follows:

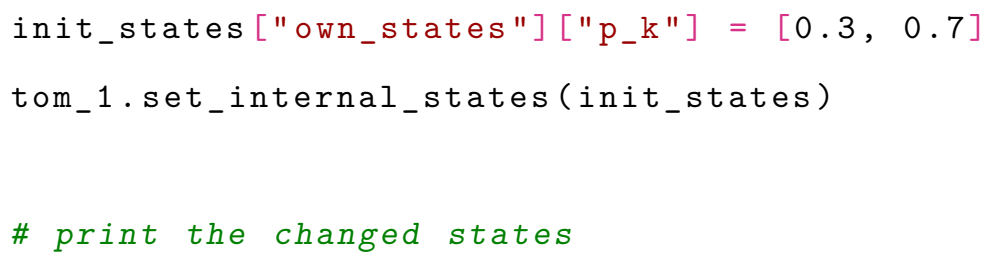


tom_1.print_internal ()

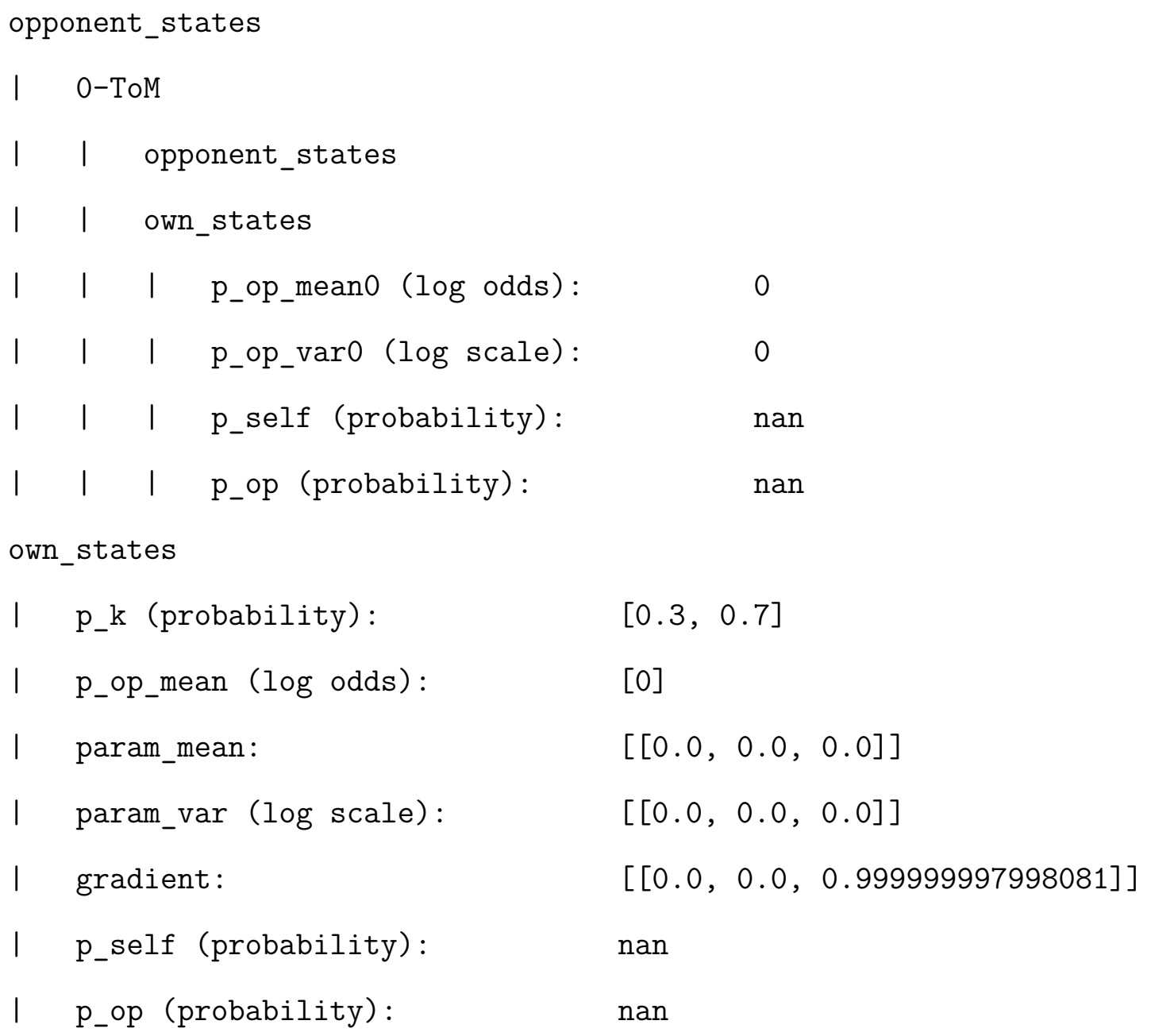

for an explanation of the internal states besides the ones detailed here we recommend you examine the help(tom_1:print_internal).

\section{Appendix B: Creating a Custom Agent}

The following includes a simple introduction on how to add and implement a new agent. The tutorial is also available and continually updated at the following link:

https://github.com/KennethEnevoldsen/tomsup/blob/master/tutorials/Creating_an_agent.ipynb 
This brief tutorial shows how to create a custom agent with a simple example.

First tomsup is imported:

import tomsup as ts

We first take a look at the current win-stay, lose-switch (WSLS) agent:

In $[8]$ : sigmund $=$ ts.WSLS() \# create agent

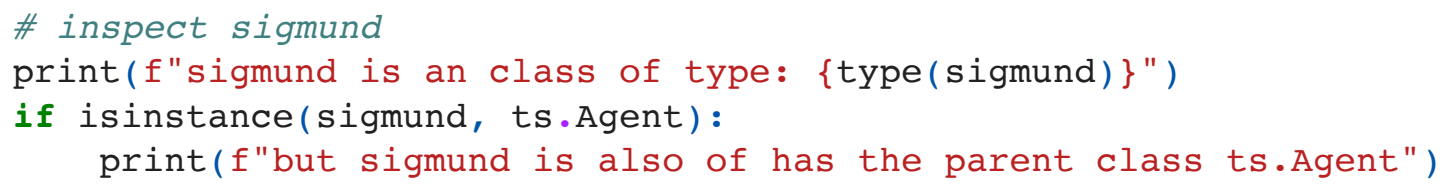

As we can see sigmund is a WSLS agent with the parent class tsAgent. This is beneficial because the WSLS inherits some of the attributes of the parent class, such as the ability to save play history and the ability to reset the agents. To see more of the inherited methods see help(ts.WSLS).

Now let's try to create our own agent, one step at a time (if you are confortable with classes in python simply jump to 'The final reversed WSLS):

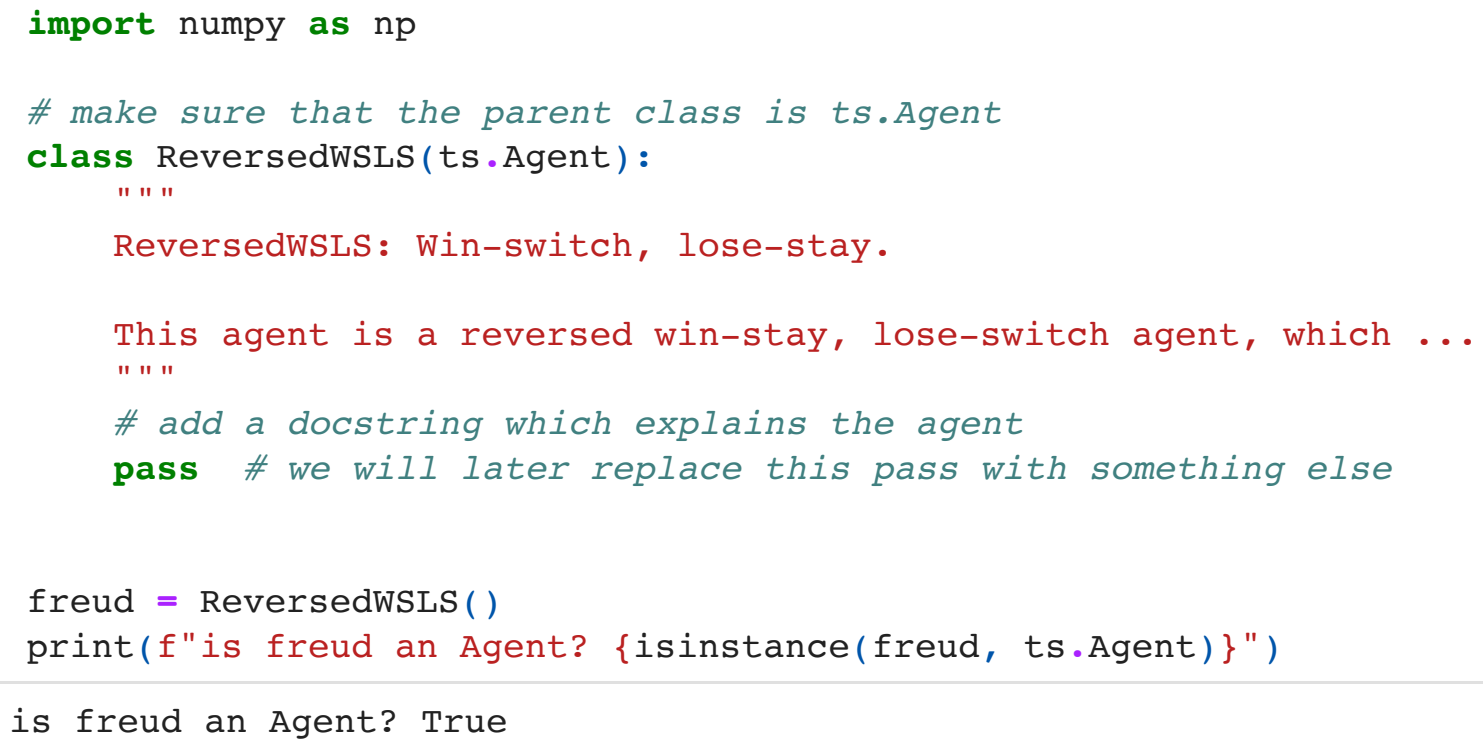

Let's add an initalization of the agent. These are things which should be created prior to the agent competing. 
class ReversedWSLS(ts.Agent):

" " "

ReversedWSLS: Win-switch, lose-stay.

This agent is a reversed win-stay, lose-switch agent, which ... " " "

def _init_(self, first_move, **kwargs): \#initalize the agent

self.strategy $=$ "ReversedWSLS" \# set the strategy name

\# set internal parameters

self.first_move = first_move

\# pass additional argument to the ts.Agent class

\# (could e.g. include 'save_history = True')

super ( ) __init__ (**kwargs)

\# save any starting parameters used when the agent is reset

self_start_params $=\{$ 'first_move': first_move, **kwargs $\}$

freud $=$ ReversedWSLS (first_move $=1$ )

print(f"what is freud's first move? \{freud.first_move\}")

print(f"what is freud's an starting parameters? \{freud.get_start_params()\}'

print(f"what is freud's strategy? \{freud.get_strategy()\}")

what is freud's first move? 1

what is freud's an starting parameters? \{'first_move': 1\}

what is freud's strategy? ReversedwSLS

In the above you sucessfully created freud as an agent and set its first move to be 1 . We also see that functions such as the get_start_params ( ) from the ts.Agent are inherited by the new agent.

Note that we have set $* *$ kwargs, this simply means that the function accepts additional arguments, e.g. save_history = True. These arguments are then passed to the super( )_init__( ), which initializes the parent class (in this case the ts.Agent class) as well as the _start_params that are the starting parameters. The starting parameters are used when resetting the agent, which is relevant e.g. when setting up a tournament.

All agents naturally need a compete function. Let us add one to the agent 
" " "

ReversedWSLS: Win-switch, lose-stay.

This agent is a reversed win-stay, lose-switch agent, which ... " " "

def _init_(self, first_move, **kwargs): \#initalize the agent self.strategy $=$ "ReversedWSLS" \# set the strategy name

\# set internal parameters

self.first_move = first_move

\# pass additional argument the ts.Agent class

\# (could e.g. include 'save_history = True')

super ( ) __init__ (**kwargs)

\# save any starting parameters used when the agent is reset self__start_params $=\{$ first_move': first_move, **kwargs

def compete(self, p_matrix, op_choice $=$ None, agent $=0$ ): " " " win-switch, lose-stay strategy, with the first move being set when the class is initilized (_ init

$\mathrm{p}$ matrix is a PayoffMatrix

op_choice is either 1 or 0

agent is either 0 or 1 and indicates the perspective of the agent in the game (whether it is player 1 or 2 )

" " "

\# if a choice haven't been made: Choose the predefined first move if self.choice is None:

\# fetch from self

self.choice = self.first_move

else: \# if a choice have been made:

\# calculate payoff from last round

payoff = p_matrix.payoff(self.choice, op_choice, agent)

\# if the agent won then switch

if payoff $==1$ :

\# save the choice in self (for next round)

\# also save any other internal states which you might

\# want the agent to keep for next round in self

self.choice = 1-self.choice

\# save action and (if any) internal states in history

\# note that add to history() is intended for later data

\# analysis, and not for use within the agent

self._add_to_history (choice = self.choice)

return self.choice \# return choice which is either 1 or 0

freud = ReversedWSLS(first_move = 1) \#create the agent

\# fetch payoff matrix for the pennygame

penny = ts.PayoffMatrix(name = "penny_competitive")

print("This is the payoffmatrix for the game (seen from freud's perspective penny( $)[0,:,:], \operatorname{sep}=" \backslash n ")$

\# have freud compete

choice = freud.compete (penny)

print(f"what is freud's choice the first round? \{choice\}")

choice = freud.compete (penny, op_choice = 1 )

print(f"what is freud's choice the second round if his opponent chose 1 ? $\{c$ 
This is the payoffmatrix for the game (seen from freud's perspective):

$\left[\begin{array}{ll}-1 & 1\end{array}\right]$

[ $1-1]$ ]

what is freud's choice the first round? 1

what is freud's choice the second round if his opponent chose 1? 1

In the above script we add freud's compete function, which on the first round choses the option specified in his starting parameters, and for future moves it uses the win-switch, lose-stay strategy. It then returns either a 0 or 1 depending on whether is chooses the right or left hand in the penny game. It is important that the agent only returns a 0 or 1 in its compete function, otherwise the agent will not function in the context of the package.

Note the self._add_to_history (choice = self. choice), which indicates which variables that should be added to the agent's history, assuming save_history is set to True. In this case we would like to save the agent's own choice.

Finally when you have the ___init__( ) and the compete( ) working you can add any additional functions you might want your agent to have. For example will we see that we have added the get_first_move ( ), which is a helper function to extract the first move of the agent.

This gives us the following finalized version of the win-switch, lose-stay agent. 


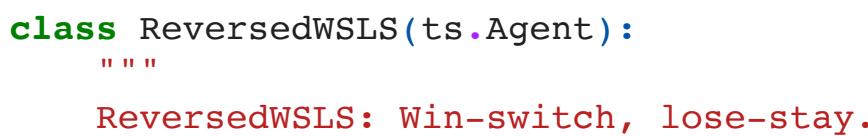

This agent is a reversed win-stay, lose-switch agent, which ...

Examples :

>> freud = ReversedWSLS(first_move $=1$ )

>> freud.compete (op_choice = None, p_matrix = penny)

1 " " "

def _init_(self, first_move, **kwargs): self.strategy $=$ "ReversedWSLS"

\# set internal parameters

self.first_move = first_move

\# pass additional argument the ts.Agent class

\# (could e.g. include 'save history = True')

super ( ) . init__ (**kwargs )

\# save any starting parameters used when the agent is reset self_start_params $=\{$ 'first_move': first_move, **kwargs $\}$

def compete(self, p_matrix, op_choice = None):

\# if a choice haven't been made: Choose the redifined first move

if self.choice is None:

self.choice = self.first move \#fetch from self

else: \# if a choice have been made:

\# calculate payoff of last round

payoff = p_matrix.payoff(self.choice, op_choice, 0 )

if payoff $==1$ : \# if the agent won then switch

\# save the choice in self (for next round)

\# also save any other internal states which you might

\# want the agent to keep for next round in self

self.choice = 1-self.choice

\# save action and (if any) internal states in history

\# note that add to history() is intended for later data

\# analysis, and not for use within the agent

self._add_to_history (choice = self.choice)

return self.choice \# return choice

\# define any additional function you wish the class should have

def get_first_move(self):

return self.first_move 


\section{Appendix C: Using tomsup for Experimental Stimuli}

The following is a simple example of how to use tomsup agents as experimental stimuli, using the software PsychoPy. The tutorial and the files required for running the experiment are available and continually updated on the following link:

https://github.com/KennethEnevoldsen/tomsup/blob/master/tutorials/psychopy_experiment 


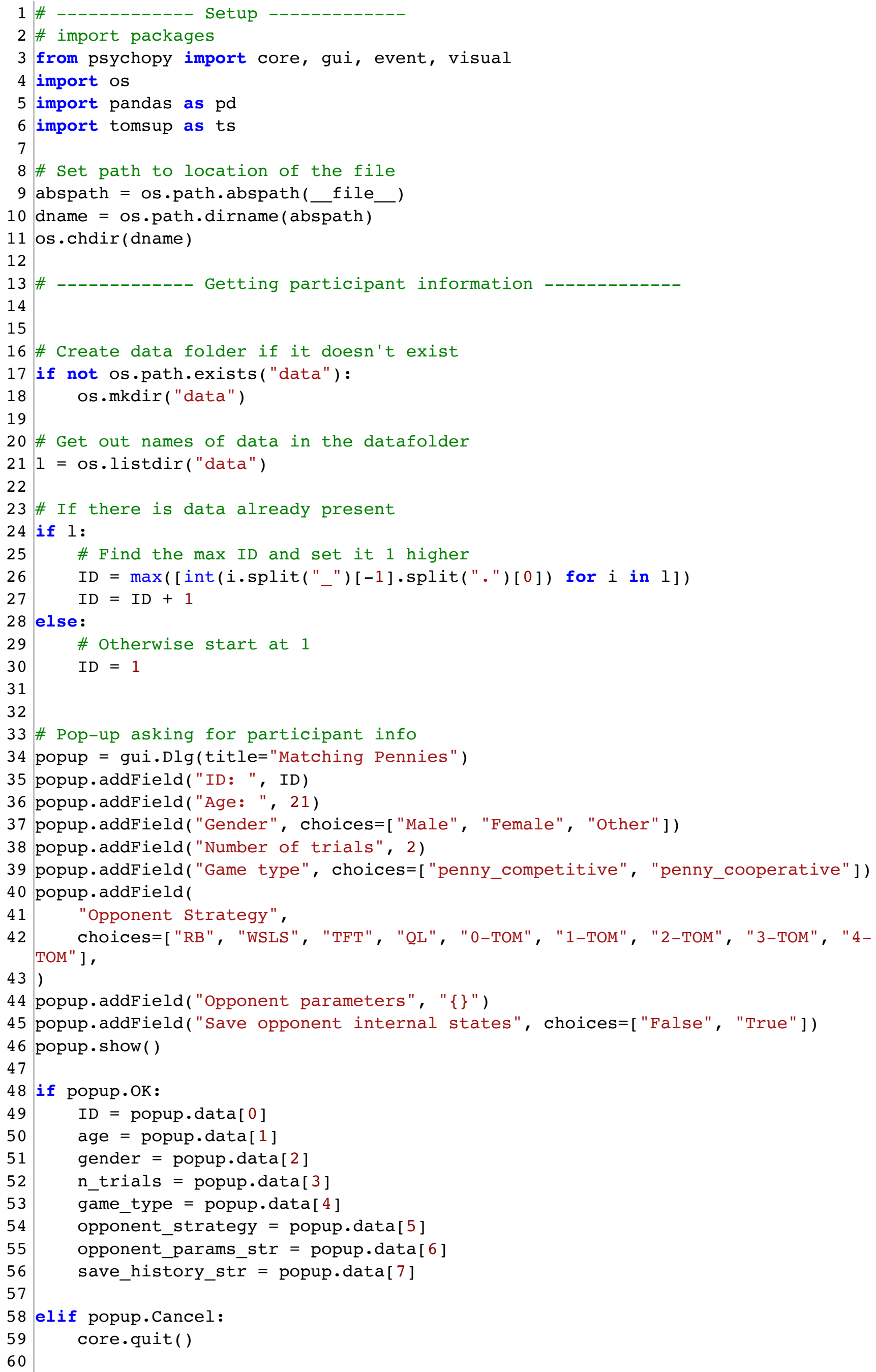


61 if save_history_str == "True":

62 save_history = True

63 else:

64 save_history $=$ False

65

66 exec (f"opponent_params $=\{$ opponent_params_str $\}$ ")

67

68 \# ------------- create agent and payoff matrix

69 opponent_params ["save_history"] = save_history

70 tom = ts.create_agents (agents=opponent_strategy, start_params=opponent_params)

71 penny $=$ ts.PayoffMatrix (name=game_type $)$

72

73 \# ----------- Defining Variables and function

74 introtext $=\mathrm{f} "$ " Dear participant

75

76 Thank you for playing against tomsup!

77 Here we will make you play against simulated agents in simple decision-making games. 78

79 We ask you for some basic demographic information. Apart from that, only performance in the game is collected.

80 If you at any time wish to do so, you are free to stop the experiment and ask for any generated data to be deleted.

81 If you have read the above and wish to proceed, press ENTER." "

82

83 rulestext_pennycompetitive = f"" "

84 You will now play a game of competitive matching pennies.

85

86 You will see the two hands of your opponent, one on the left, the other on the right.

87 One of the hands hides a penny. Your goal is to figure out which of the two hands contain the penny.

88 If you guess the correct hand, you get a point and your opponent loses a point.

89 If you guess incorrectly, you lose a point and your opponent gains a point.

90 The game will last for $\{$ n_trials $\}$ trials.

91

92 By pressing the "right arrow" on your keyboard, you guess "right".

93 By pressing the "left arrow" on your keyboard, you guess "left".

94 After guessing, press ENTER to continue.

95 To quit the game, press ESCAPE.

96 When you have read and understood the above, press ENTER to continue." "

97

98 rulestext_pennycooperattive = f" "

99 You will now play a game of cooperative matching pennies.

100

101 You will see the two hands of your opponent, one on the left, the other on the right.

102 One of the hands hides a penny. Your goal is to figure out which of the two hands contain the penny.

103 If you guess the correct hand, you and your opponent both get get a point.

104 If you guess incorrectly, you and your opponent both lose a point.

105 The game will last for $\left\{n_{\text {_trials }}\right.$ trials.

106

107 By pressing the "right arrow" on your keyboard, you guess "right".

108 By pressing the "left arrow" on your keyboard, you guess "left".

109 After guessing, press ENTER to continue.

110 To quit the game, press ESCAPE.

111 When you have read and understood the above, press ENTER to continue.""

112

113 \# Set rulestext to fit the specified game

114 if game_type == "penny_competitive":

115 rulestext $=$ rulestext_pennycompetitive

116 elif game_type == "penny_cooperative": 
119 \# Show_text for normal text

120 def show_text(txt):

$121 \mathrm{msg}=\mathrm{visual}$. TextStim(win, text=txt, height=0.05)

122 msg.draw()

123 win.flip()

131 \# setting window and reading images

132 win $=$ visual. Window $($ fullscr $=$ False $)$

133 stopwatch $=\operatorname{core} \cdot \operatorname{Clock}()$

134 RH_closed = "images/RH_closed.png"

135 LH_closed = "images/LH_closed.png"

136 LH_open = "images/LH_open.png"

137 RH_open = "images/RH_open.png"

138 LH_coin = "images/LH_coin.png"

139 RH_coin = "images/RH_coin.png"

$$
\{
$$

\section{0}

161 \# run intro

162 show_text (introtext)

163 show_text (rulestext)

164 op_choice $=$ None $\#$ setting opponent choice to none for the first round

165 current_score_part $=0$

166 current_score_tom $=0$

167

168 img_pos $1=[-0.50,0.0]$

169 img_pos $2=[0.50,0.0]$

170 img_size $=[0.9,0.9]$ 


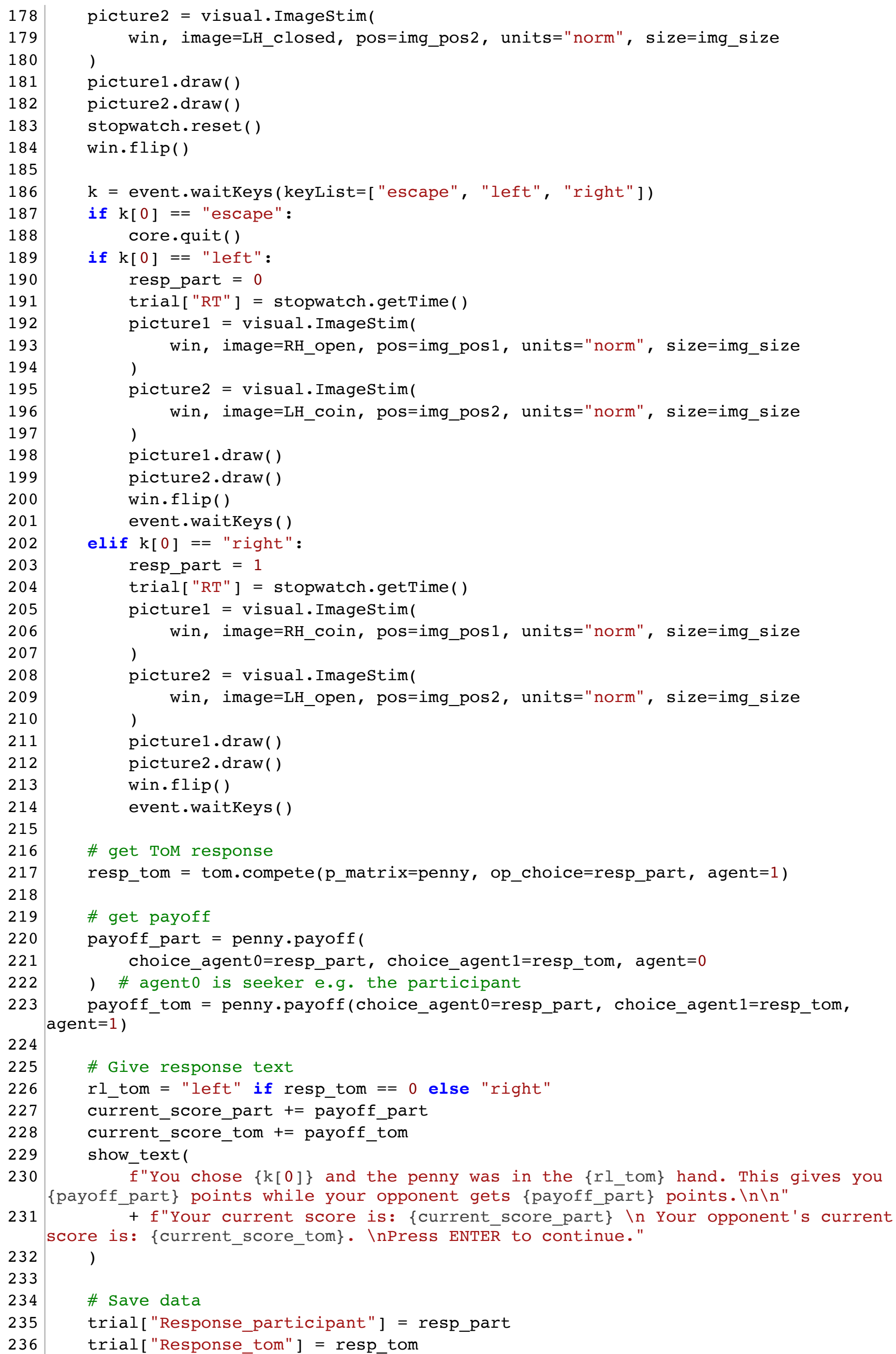


237 trial["payoff_participant"] = payoff_part

238 trial["payoff_tom"] = payoff_tom

239 if save_history:

240 trial ["tom_internal_states"] = tom.get_internal_states()

\# write data (writes at each trial, so that even if the program crashes there should be an issue)

243 pd.DataFrame(trial_list).to_csv("data/ID_" + str(ID) + ".CSV")

245 \# write data

246 pd.DataFrame(trial_list).to_csv("data/ID_" + str(ID) + ".CSV")

247

248 show_text (

249 " " "

250 This concludes the game!

251 Thank you playing!

252

253 Press ENTER to quit.

254 " " "

$255)$

256

257 event.waitKeys ()

258

259 \# Close psychopy

260 core.quit( ) 


\section{Appendix D: Comparison with the VBA Implementation}

We here briefly compare the behaviour and speed of tomsup with that of the $k-T o M$ implementation in the VBA package for MATLAB. Code for reproducing the comparison can be found at https://github.com/KennethEnevoldsen/tomsup/papers/introducing_tomsup/comparison. This includes a behavioral test, which validates that tomsup k-ToM agents produce the same behaviour as the VBA implementation. To test this a random-bias agent competed a k-ToM with $k \in\{0,1,2\}$ with the same starting parameters. Both agents were run as implemented, but with their choices forced to a random sample of 10 trials, which were kept the same across the tomsup and VBA implementention. At each round the internal states was found to only differ after the 16th decimal place.

The folder also includes a speed test, which compares the efficiency of the two implementations. In the speed test, a Random Bias agent was made to play against various kinds of opponents for 8 simulations of 60 trials. This was repeated 20 times. In Table 2 , the mean time across the 20 tests and the standard deviation is shown. It can be seen that the tomsup implementation is consistently faster, even without parallelization.

\section{Table 2}

\section{Implementation speed}

\begin{tabular}{||c|ccc||}
\hline agent & VBA & tomsup & tomsup 4 cores \\
\hline \hline RB & $0.33(0.03)$ & $0.003(0.0002)$ & $0.04(0.15)$ \\
\hline 0-ToM & $0.53(0.03)$ & $0.008(0.0002)$ & $0.05(0.17)$ \\
\hline 1-ToM & $0.80(0.07)$ & $0.04(0.0005)$ & $0.05(0.15)$ \\
\hline 2-ToM & $1.70(0.10)$ & $0.22(0.002)$ & $0.10(0.16)$ \\
\hline 3-ToM & $4.42(0.15)$ & $1.07(0.004)$ & $0.33(0.15)$ \\
\hline 4-ToM & $13.08(1.31)$ & $5.37(0.03)$ & $1.53(0.15)$ \\
\hline 5-ToM & $39.05(0.69)$ & $27.01(0.05)$ & $7.30(0.14)$ \\
\hline
\end{tabular}

